\title{
Addiction to DUSP1 protects JAK2V617F-driven polycythemia vera progenitors against inflammatory stress and DNA damage, allowing chronic proliferation
}

\author{
J. Stetka $\oplus^{1} \cdot$ P. Vyhlidalova $\mathbb{1}^{1,2} \cdot$ L. Lanikova ${ }^{1,3} \cdot$ P. Koralkova ${ }^{1} \cdot$ J. Gursky $\mathbb{1}^{4} \cdot$ A. Hlusi ${ }^{5} \cdot$ P. Flodr ${ }^{6} \cdot$ S. Hubackova ${ }^{7}$. \\ J. Bartek ${ }^{4,8,9,10} \cdot$ Z. Hodny ${ }^{9} \cdot$ V. Divoky ${ }^{1,5}$
}

Received: 10 February 2019 / Revised: 20 March 2019 / Accepted: 21 March 2019 / Published online: 9 April 2019

(c) The Author(s) 2019. This article is published with open access

\begin{abstract}
Inflammatory and oncogenic signaling converge in disease evolution of BCR-ABL-negative myeloproliferative neoplasms, clonal hematopoietic stem cell disorders characterized by gain-of-function mutation in JAK2 kinase (JAK2V617F), with highest prevalence in patients with polycythemia vera (PV). Despite the high risk, DNA-damaging inflammatory microenvironment, PV progenitors tend to preserve their genomic stability over decades until their progression to post-PV myelofibrosis/acute myeloid leukemia. Using induced pluripotent stem cells-derived $\mathrm{CD} 34^{+}$progenitor-enriched cultures from JAK2V617 ${ }^{+}$PV patient and from JAK2 wild-type healthy control, CRISPR-modified HEL cells and patients' bone marrow sections from different disease stages, we demonstrate that JAK2V617F induces an intrinsic IFN $\gamma$ - and NF-kBassociated inflammatory program, while suppressing inflammation-evoked DNA damage both in vitro and in vivo. We show that cells with JAK2V617F tightly regulate levels of inflammatory cytokines-induced reactive oxygen species, do not fully activate the ATM/p53/p21waf1 checkpoint and p38/JNK MAPK stress pathway signaling when exposed to inflammatory cytokines, suppress DNA single-strand break repair genes' expression yet overexpress the dual-specificity phosphatase (DUSP) 1. RNAi-mediated knock-down and pharmacological inhibition of DUSP1, involved in p38/JNK deactivation, in HEL cells reveals growth addiction to DUSP1, consistent with enhanced DNA damage response and apoptosis in DUSP1inhibited parental JAK2V617F ${ }^{+}$cells, but not in CRISPR-modified JAK2 wild-type cells. Our results indicate that the JAK2V617F ${ }^{+}$PV progenitors utilize DUSP1 activity as a protection mechanism against DNA damage accumulation, promoting their proliferation and survival in the inflammatory microenvironment, identifying DUSP1 as a potential therapeutic target in PV.
\end{abstract}

These authors contributed equally: J. Stetka, P. Vyhlidalova

Supplementary information The online version of this article (https:// doi.org/10.1038/s41388-019-0813-7) contains supplementary material, which is available to authorized users.

\section{J. Bartek}

jb@cancer.dk

$\bowtie$ Z. Hodny

zdenek.hodny@img.cas.cz

$\triangle$ V. Divoky

vladimir.divoky@upol.cz

Extended author information available on the last page of the article.

\section{Introduction}

Inflammatory signaling is a common mechanism fueling genotoxic stress and contributing to tumorigenesis including transformation in the hematopoietic system [1]. Three subtypes of myeloproliferative neoplasms (MPNs): polycythemia vera $(\mathrm{PV})$, essential thrombocythemia (ET), and primary myelofibrosis (PMF), are clonal hematological disorders with overlapping phenotypes characterized by oncogenic Janus kinase (JAK)-signal transducers and activators of transcription (STAT)-mediated signaling, associated chronic inflammation, long clinical course, and long-term disease evolution [2]. The most frequent MPN driving mutation, the gain-of-function JAK2V167F, activates type-1 myeloid cytokine receptors and is detected in $>95 \%$ of PV and 50-60\% ET and PMF as an early somatic event [3]. Dysregulation of this key cytokine receptor signaling fuels formation of a bone marrow (BM) 
microenvironment with aberrant synthesis of inflammatory cytokines and chemokines, triggering persistent, systemic inflammatory response marked by elevated inflammatory markers in circulation $[4,5]$. In a mouse model, inflammatory factors contribute to remodeling of the BM microenvironment into leukemic niche that inhibits normal hematopoiesis and favors leukemic stem cell formation and myelofibrosis [6, 7]. However, despite the pronounced chronic inflammation, the two "proliferative" MPN disease types, PV and ET, with overproduction of mature, functional myeloid elements, have a relatively low cumulative incidence of blast transformation and fibrotic progression [8]. We therefore hypothesized that the JAK2V617F-driven activation of cell autonomous and non-cell autonomous inflammatory programs induces some feedforward protective loop that guards PV progenitors from cell-intrinsic and cell-extrinsic DNA-damaging stimuli, providing a barrier that prevents cell cycle arrest, delays stressinduced regulatory hematopoietic circuits associated with myelofibrosis $[9,10]$, and decreases DNA damage accumulation associated with rapid malignant transformation [11].

Human induced pluripotent stem cells (iPSCs) have been widely used for disease modeling, including PV [12], and transcriptional profiling, cell cycle, and DNA damage response (DDR) studies in specialized cell types differentiated from iPSCs [13]. Here, we compared differentiated, PV patient-specific iPSCs with a homozygous JAK2V617F constitution and gender-matched iPSCs with wild-type JAK2 (JAK2wt), along with genetically modified JAK2V617F-expressing HEL cells and immunohistochemical (IHC) staining of PV patients' BM, to investigate the hierarchy and consequences of the activated inflammatory signature. We found an increased reactive oxygen species (ROS)-buffering capacity and overexpression of the dual-specificity phosphatase (DUSP) 1 in JAK2V617F cells as candidate mechanisms keeping fast-cycling PV progenitors in a proliferative phase despite the surrounding genotoxic inflammatory microenvironment.

\section{Results}

\section{Validation of the iPSC-derived hematopoietic differentiation model}

PV patient-specific JAK2V617F ${ }^{+}$(PVB1.4) and control JAK2wt (BXS0116) iPSC lines, reprogrammed from BM CD $34^{+}$cells, were differentiated for 9 days (hereafter d9) into $\mathrm{CD}^{+} 4^{+}$hematopoietic progenitors (Supplementary Materials and Methods) [14]. Contaminating cell types from other than hematopoietic lineages were present, however, our protocol generated cultures with comparable abundance of $\mathrm{CD} 34^{+}$hematopoietic progenitors in both JAK2wt and
V617F $\mathrm{F}^{+}$-differentiated cultures (hereafter referred to as $\mathrm{CD} 34^{+}$progenitor-enriched cultures, P-ECs), with comparable abundance of other key hematopoietic surface antigens and transcripts for early hematopoietic markers and signaling pathways regulating stem cell pluripotency (Supplementary Fig. 1a-d). As expected, contrary to JAK2wt cells, the JAK2V617F ${ }^{+} \mathrm{CD}_{4}{ }^{+}$P-ECs at $\mathrm{d} 9$ exhibited markers of myeloid/erythroid lineage biased differentiation (Supplementary Fig. 1e). Furthermore, both PVB1.4 and BXS0116 iPSC lines showed unimpaired activation of DDR markers in both JAK2wt and JAK2V617F ${ }^{+}$undifferentiated iPSC clones following DNA-damaging X-ray irradiation (Supplementary Fig. 1f), suggesting functionality of DDR signaling in our model. Next, we treated the model cell lines with interferon- $\gamma$ (IFN $\gamma$ ), tumor necrosis factor-alpha (TNF $\alpha$ ), and transforming growth factor-beta 1 (TGF $\beta 1$ ), the upstream principal regulators of the inflammatory program and microenvironment alterations in MPN [15-17]. IFN $\gamma$, $\mathrm{TNF} \alpha$, and TGF $\beta 1$ are known to induce genotoxic stress, DDR signaling and senescence in both normal and tumor cells [18] and we hypothesized that the JAK2V617F ${ }^{+} \mathrm{P}$ ECs are refractory to such treatment.

\section{$\mathrm{JAK}^{2} \mathrm{~V} 17 \mathrm{~F}^{+}$progenitors activate an inflammatory signature intrinsically by wake of IFN $\gamma$ transcription and IFNY-dependent STAT1 signaling}

Subpopulations of hematopoietic stem cells (HSCs) and progenitors exhibit high IFN responsiveness maintained by intrinsic expression of a subset of IFN-stimulated genes [19, 20]. As the JAK2V617F ${ }^{+}$cells display high STAT1 activation $[15,21]$ and the most highly, cell-autonomously induced cytokine in JAK2V617F ${ }^{+}$progenitors is CXCL10 [22, 23], an IFN $\gamma$-inducible chemokine [24], we first tested intrinsic and inflammatory cytokine-inducible expression of IFN $\gamma$ in $\mathrm{JAK} 2 \mathrm{~V} 617 \mathrm{~F}^{+}$progenitors. D9 derivatives of the JAK2V617F ${ }^{+}$cultures intrinsically expressed $I F N G$, which was further increased after treatment with inflammatory cytokines (IFN $\gamma, \mathrm{TNF} \alpha$, and TGF $\beta 1$ treatment of $\mathrm{d} 9 \mathrm{CD} 34^{+}$ P-ECs for $24 \mathrm{~h}$, hereafter d9 cyt) specifically in the $\mathrm{JAK} \mathrm{V}_{617 \mathrm{~F}^{+}} \mathrm{CD}^{+} 4^{+}$P-ECs but not in their JAK2wt counterparts (Fig. 1a). IFN $\gamma$-producing immature myeloid cells were present in BM at all PV disease stages (PV, MF1/2 PV with fibrosis grade 1 and 2, MF-3 PV with fibrosis grade 3/post-PV MF) [25] (Fig. 1b). IFN $\gamma$ staining also colocalized with the CD34 antigen in embryoid bodies (EBs) derived from the JAK2V617F ${ }^{+}$iPSCs (Supplementary Fig. 1g). STAT1 phosphorylation on tyrosine 701 was induced by IFN $\gamma$ alone or combined with TGF $\beta 1$ and/or $\mathrm{TNF} \alpha$ more robustly in the $\mathrm{JAK}_{2} \mathrm{~V}_{617 \mathrm{~F}^{+}} \mathrm{CD} 34^{+} \mathrm{P}$-ECs than in the JAK2wt cells (Supplementary Fig. 1h). 
a

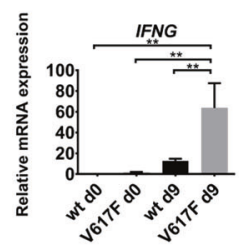

C

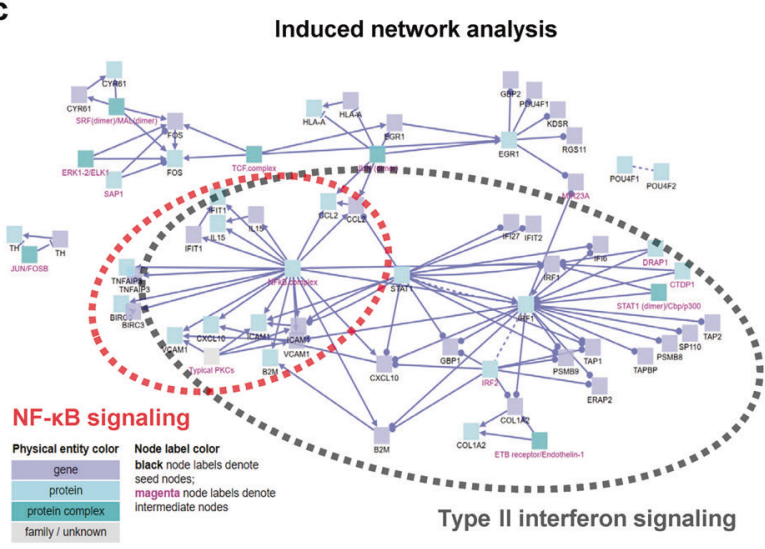

b

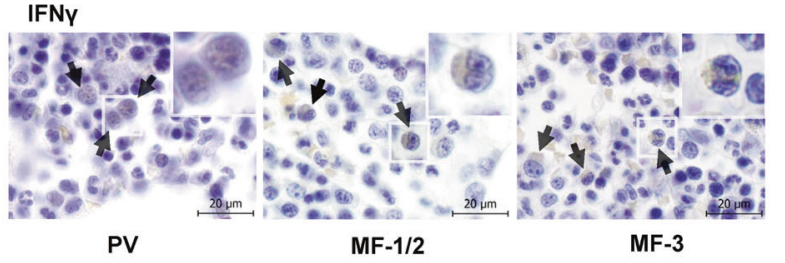

d

Type II interferon signaling

ES: 0.50

NES: 3.48

P: $<0.001$

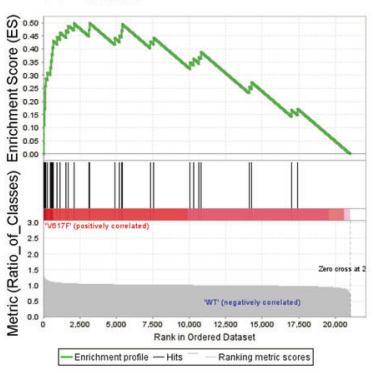

NES: 2.53

$P:<0.001$

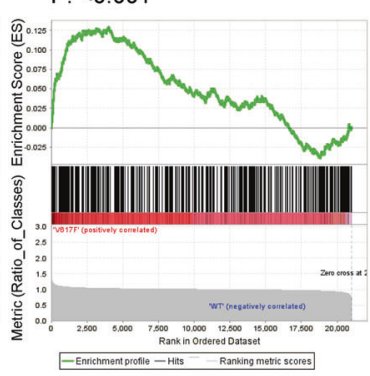

e

Type II interferon signaling

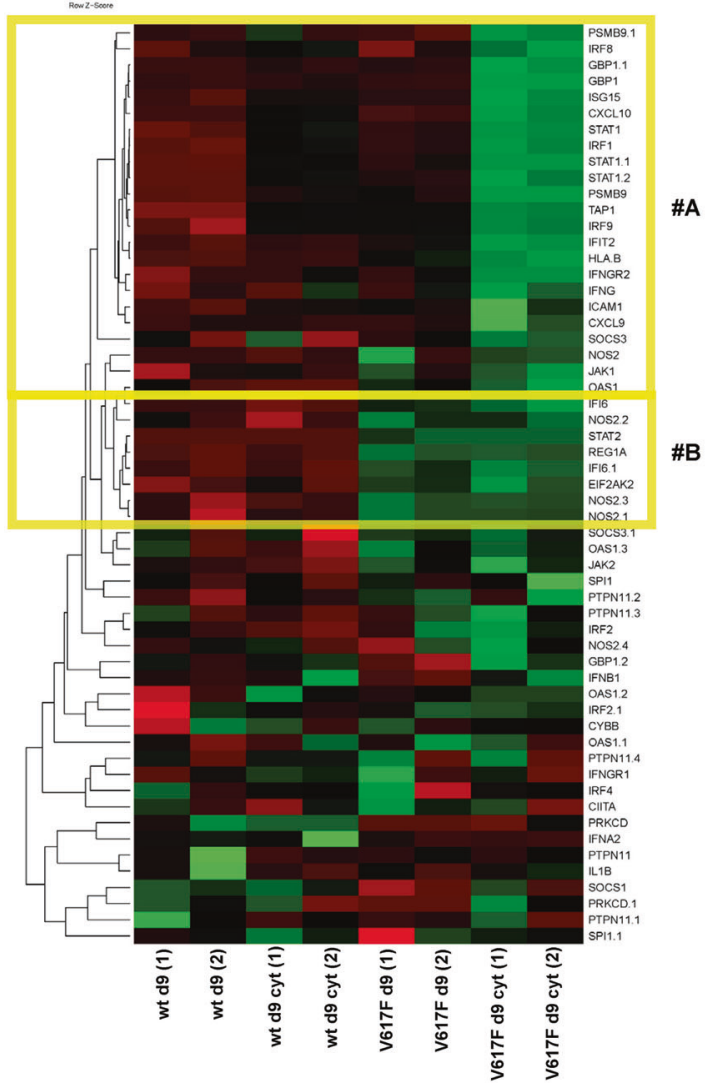

To gain more insights into the expression program differentially regulated between $\mathrm{d} 9 \mathrm{JAK} 2 \mathrm{wt}$ and $\mathrm{JAK} 2 \mathrm{~V} 617 \mathrm{~F}^{+}$ CD $34^{+} \mathrm{P}-\mathrm{EC}$, in medium without or with inflammatory cytokines, we performed whole-genome transcriptional
CXCL9

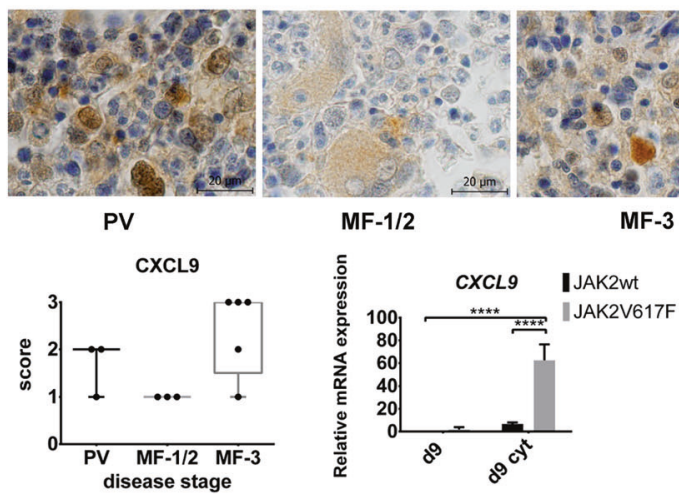

CXCL10
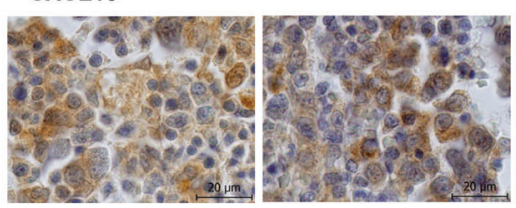

PV

MF-1/2

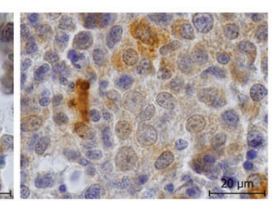

CXCL10
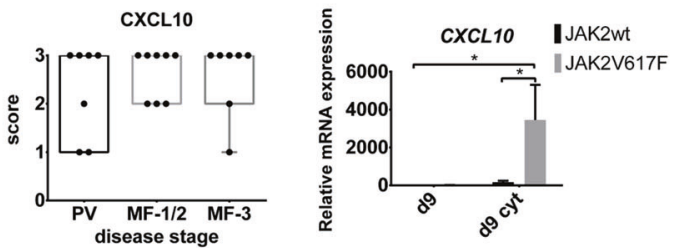

profiling. Top 20 differentially upregulated genes included overexpression of type II (IFN $\gamma$ )-stimulated genes in the JAK2V617F ${ }^{+}$versus the JAK2wt P-ECs (Supplementary Fig. 1i). Among the affected networks identified using the 
Fig. 1 Intrinsic and proinflammatory cytokine-induced expression of IFN $\gamma$ and IFN $\gamma$-regulatory program in $\mathrm{JAK} 2 \mathrm{~V} 617 \mathrm{~F}^{+}$progenitors. a Left bar graph: intrinsic IFNG mRNA expression (mean $\pm \mathrm{SD} ; n=3$ independent experiments) in $\mathrm{d} 9 \mathrm{JAK} 2 \mathrm{~V} 617 \mathrm{~F}^{+} \mathrm{CD} 34^{+} \mathrm{P}-\mathrm{EC}$ compared with d9 JAK2wt CD34 ${ }^{+}$P-ECs and with undifferentiated JAK2wt and $\mathrm{V} 617 \mathrm{~F}^{+}$iPSC lines (d0). Right bar graph: IFNG mRNA expression in three independent experiments after treatment with IFN $\gamma, \mathrm{TNF} \alpha$, and TGF $\beta 1$ inflammatory cytokines (d9 cyt). $* P \leq 0.05$, $* * P \leq 0.01, * * * P \leq 0.001$, one-way and two-way ANOVA, respectively. b Representative IHC staining of BM sections for IFN $\gamma$ among different disease stages of PV progression. Scale bar, $20 \mu \mathrm{m}$. Insets in each panel show magnification of individual IFN $\gamma$-producing cells. c Visualization of induced network module analysis made by ConsensusPathDB using differentially upregulated genes $(\log \mathrm{FC}>1, q<$ 0.1 cut-off) between JAK2wt and $\mathrm{V}^{2} 17 \mathrm{~F}^{+} \mathrm{CD} 34^{+}$P-ECs treated with inflammatory cytokines (d9 cyt) as seed genes with seed nodes (black) and intermediate nodes (magenta) connected through functional and physical links. Red circle depicts visualized nodes associated with NF$\kappa \mathrm{B}$ pathway, with significant type II interferon signaling genes overlap (gray circle). d Left: GSEA plot of type II interferon signaling gene set members (WikiPathway, $n=37$ ) in d9 cyt JAK2V617F ${ }^{+}$and JAK2wt $\mathrm{CD}_{3} 4^{+}$P-ECs. Right: GSEA of d9 cyt JAK2V617F ${ }^{+}$and JAK2wt CD34 ${ }^{+}$P-ECs using a set of 424 NF- $\mathrm{KB}$ target genes (http://www.bu. edu/nf-kb/gene-resources/target-genes/). e Heatmap representation of differential expression of type II interferon signaling gene set members in d9 JAK2wt and JAK2V617F ${ }^{+} \mathrm{CD}^{+} 4^{+}$P-ECs, untreated (d9) or treated with inflammatory cytokines (d9 cyt). Columns in the heatmap represent individual samples in experimental duplicates $(1,2)$. f IHC staining of CXCL9 and CXCL10 in representative BM sections from patients along the progression of PV. Scale bar, $20 \mu \mathrm{m}$. Boxplots show quantification of the number of cells expressing CXCL9 and CXCL10 in sections of patients from grouped disease stages. The bar graphs show mRNA expression (mean $\pm \mathrm{SD}$ ) of three independent experiments for $C X C L 9$ and $C X C L 10$ in d9 cyt JAK2V617F ${ }^{+}$CD $34^{+}$P-ECs, compared with untreated (d9) or treated (d9 cyt) JAK2wt CD34 ${ }^{+} \mathrm{P}-$ ECs. $* P \leq 0.05, * * * * P \leq 0.0001$, Mann-Whitney test (IHC) and twoway ANOVA (qRT-PCR). See also Supplementary Fig. 1

ConsensusPathDB pathway analysis, IFN $\gamma$ and nuclear factor-kappa $\mathrm{B}(\mathrm{NF}-\kappa \mathrm{B})$ signaling pathways were enriched for differentially overexpressed genes in $\mathrm{d} 9$ cyt JAK2V617F ${ }^{+} \mathrm{CD}^{+}{ }^{+}$P-ECs as compared with JAK2wt d9 cyt cells (Fig. 1c and Supplementary Fig. 1j). IFN $\gamma$ and NF$\kappa \mathrm{B}$ signaling gene set members were significantly upregulated in $\mathrm{d} 9$ cyt JAK2V617F ${ }^{+}$compared with JAK2wt CD34 + P-ECs (determined by gene set enrichment analysis (GSEA), Fig. 1d). An expression heatmap for IFN $\gamma$ signaling genes showed two clusters: cluster \#A with genes exclusively induced in the $\mathrm{JAK} 2 \mathrm{~V} 617 \mathrm{~F}^{+}$cells upon treatment with inflammatory cytokines, and cluster \#B containing intrinsically upregulated IFN $\gamma$-dependent genes in the JAK2V617F ${ }^{+}$cells (Fig. 1e). These experiments suggested cell-intrinsic IFN $\gamma$ priming driven by JAK2V617F.

\section{Cooperation of IFN $\gamma$, TNF $\alpha$ and/or TGF $\beta 1$ triggers robust expression of pro-fibrogenic chemokines in JAK2V617F ${ }^{+}$CD34 $^{+}$P-ECs}

Among the most differentially upregulated NF- $\kappa \mathrm{B}$ target genes (Supplementary Fig. 1k) in the d9 cyt JAK2V617F
$+\mathrm{CD} 34^{+}$P-ECs, which overlapped with the induced IFN $\gamma$ signaling gene set members were pro-fibrogenic chemokines CXCL9 and CXCL10. Both chemokines were highly expressed in PV patients' samples; the level of CXCL9 was highest in progressed post-PV MF-3 while CXCL10 was expressed constitutively across disease stages (Fig. 1f). We tested whether overexpression of these chemokines in the JAK2V617F ${ }^{+}$P-ECs is mainly due to IFN $\gamma$ responsiveness (or other individual cytokines) of cells exhibiting an intrinsic IFN $\gamma$ priming state or it reflects a cooperation between IFN $\gamma$ and upstream pro-fibrogenic regulators TNF $\alpha$ and TGF $\beta 1$ [24, 26-28]. In vivo, consistently with earlier studies [29, 30], we detected progressively increased expression of TNF $\alpha$ with staining restricted to fibrotic tissue and constitutive expression of TGF $\beta 1$ during the disease progression (Supplementary Fig. 11). While IFN $\gamma, \mathrm{TNF} \alpha$, or TGF $\beta 1$ used alone did not elicit any significant pro-fibrogenic response (data not shown), combinations of IFN $\gamma$ with $\mathrm{TNF} \alpha$ and IFN $\gamma$ with $\mathrm{TNF} \alpha$ and TGF $\beta 1$ robustly induced CXCL9 and CXCL10 in the JAK2V617F ${ }^{+}$progenitors (Fig. 1f). As IL6 and CCL3 were also shown to be an important part of the proinflammatory profile in patients with MPN [5], we have analyzed their expression in BM sections from patients at different PV disease stages. Both cytokines were constitutively present across the disease stages with high expression of CCL3 in basophillike progenitor cells, as previously described in CML [31], and modest expression of IL6 (Supplementary Fig. 11).

These results supported a powerful pro-fibrogenic response of the $\mathrm{JAK} 2 \mathrm{~V} 617 \mathrm{~F}^{+}$progenitors and a cooperation of inflammatory mediators in disease evolution, including the role of CXCL10 and CXCL9 in fibrogenesis.

\section{JAK2V617F-mediated protection against inflammation-evoked DNA damage accumulation and the DDR}

Although some studies reported abundant JAK2V617Fdependent oxidative DNA lesions due to enhanced ROS generation [32] and increased homologous recombination (HR) activity and genetic instability fueled by the oncogenic JAK2V617F in MPN [33], others have questioned such features of the JAK2V617F-expressing progenitors [34]. To establish whether and how JAK2V617F causes oncogenic stress and influences sensitivity or resistance to inflammation-evoked DNA damage, we first analyzed the overall degree of DDR activation in BM sections from patients at different $\mathrm{PV}$ disease stages. Oxidative DNA damage (8-oxoguanine, 8-oxoG) was barely detectable in $\mathrm{PV}$ and MF-1/2, and was only increased in post-PV MF-3, due to positivity of megakaryocytes (Fig. 2a and 
a
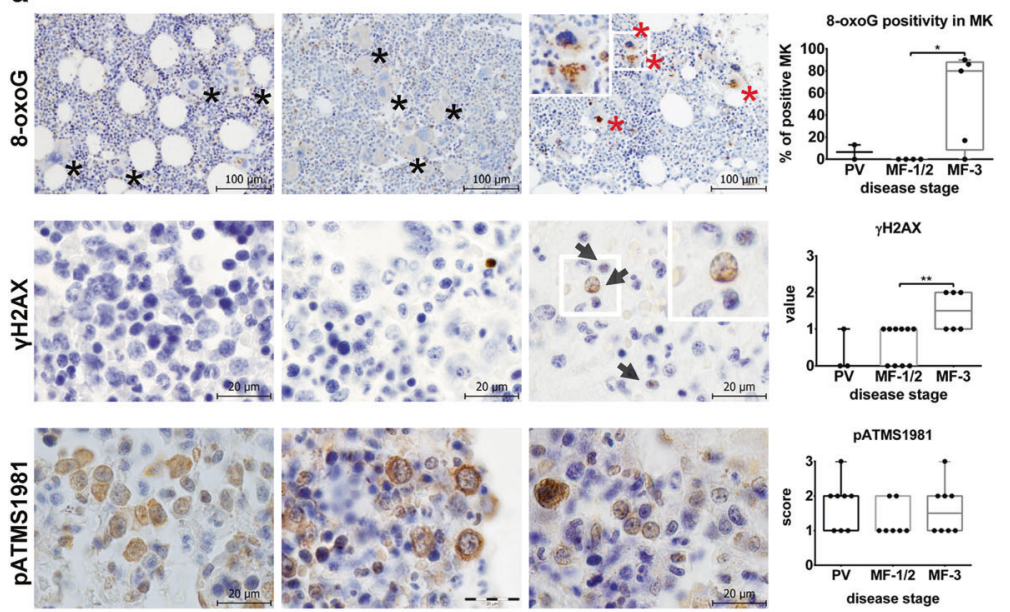

$\gamma \mathrm{H} 2 \mathrm{AX}$
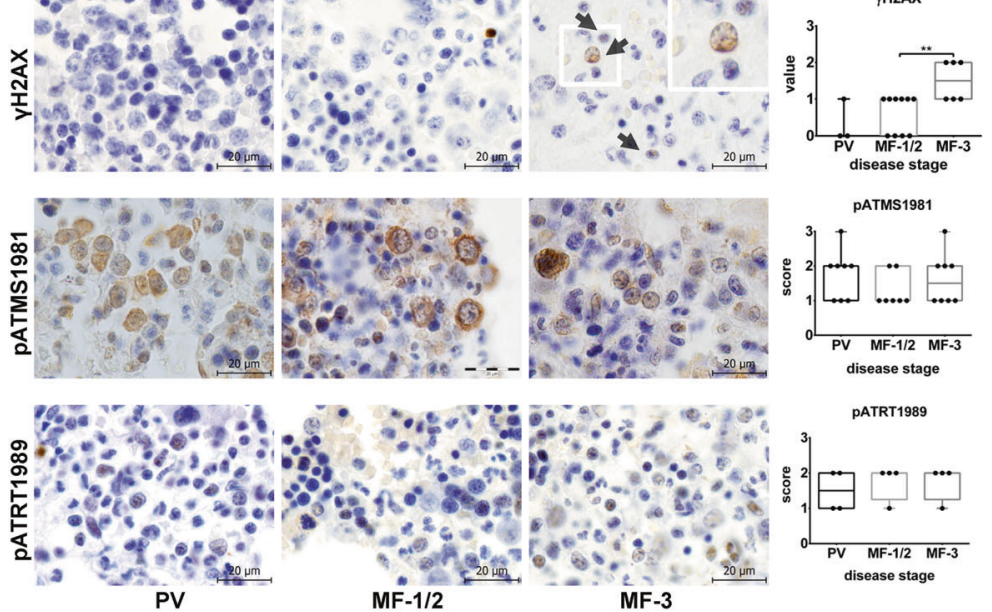

MF-3

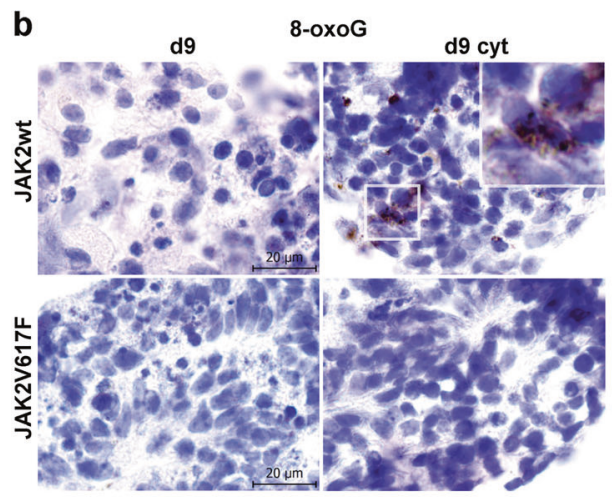

C
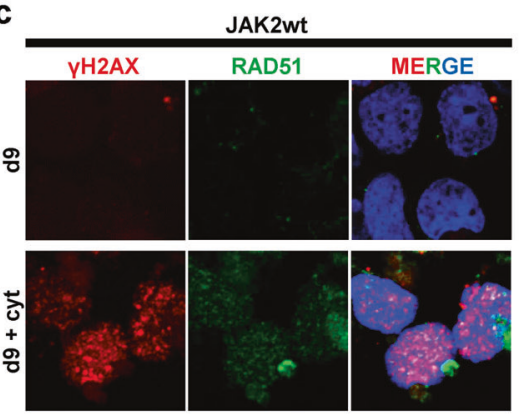

$\gamma \mathrm{H} 2 \mathrm{AX}$

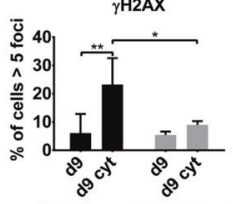

IJAK2Wt |IJAK2V617F
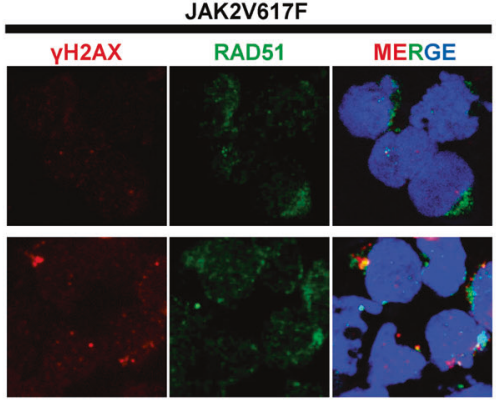

nuclear RAD51

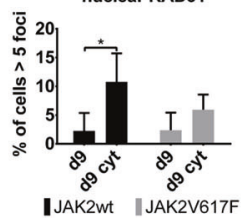

d

DNA single-strand break repair genes ES: -0.20

NES: -2.59

$P:<0.001$

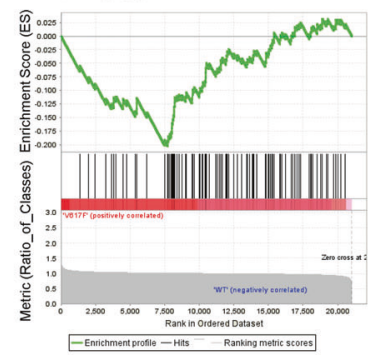

DNA single-strand break repair genes (1)

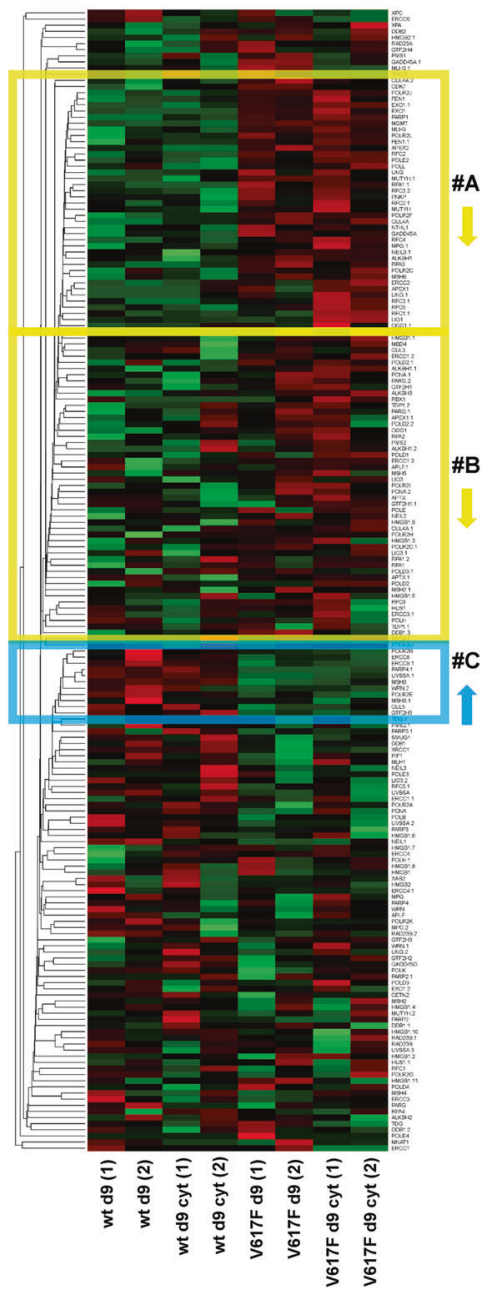

Supplementary Fig. 2a). The global DDR marker, Ser 139phosphorylated histone $\mathrm{H} 2 \mathrm{AX}(\gamma \mathrm{H} 2 \mathrm{AX})$, showed similar patterns, with moderate positive staining only in post-PV MF-3 samples (Fig. 2a). The activated form of ATM, Ser 1981-phosphorylated ATM (pATMS1981), was constitutively present in cytoplasm in all disease stages, consistent with ROS-mediated activation [35], whereas the activated form of ATR, Thr 1989-phosphorylated ATR 
Fig. 2 Protection against inflammation-induced DNA damage accumulation and DDR in PV cells. a Immunohistochemistry staining for oxidative DNA damage and DDR markers in PV, MF-1/2, and postPV MF-3 patients. In 8-oxoguanine (8-oxoG) staining, red asterisks mark 8-oxoG-positive megakaryocytes (MKs), black asterisks denote negative MKs; scale bar, $100 \mu \mathrm{m}$. In $\gamma \mathrm{H} 2 \mathrm{AX}$ staining, inset in MF-3 panel shows magnification of cells stained positive for $\gamma \mathrm{H} 2 \mathrm{AX}$ foci; scale bar, $20 \mu \mathrm{m}$. pATMS1981 staining revealed cytoplasmic and pATRT1989 nuclear positivity; scale bars, $20 \mu \mathrm{m}$. Boxplots show quantification of the number of cells expressing indicated markers in sections of patients from grouped disease stages. $* P \leq 0.05$, ** $P \leq$ 0.01, Mann-Whitney test. b Immunostaining for 8-oxoG of paraffinembedded EBs derived from d9 and d9 cyt JAK2wt and V617F iPSCs. c $\gamma \mathrm{H} 2 \mathrm{AX}$ (red) and RAD51 (green) staining of $\mathrm{d} 9$ and $\mathrm{d} 9 \mathrm{cyt}$ JAK2wt and JAK2V617F ${ }^{+}$CD34 ${ }^{+}$P-ECs. Charts show percentage of cells \pm SD in three independent experiments with more than five $\gamma \mathrm{H} 2 \mathrm{AX}$ or RAD51 foci per cell in nucleus. $* P \leq 0.05$, $* * P \leq 0.01$, twoway ANOVA. d Top: GSEA of d9 cyt JAK2V617F ${ }^{+}$and JAK2wt $\mathrm{CD}^{+} 4^{+} \mathrm{P}-\mathrm{EC}$ s using a set of 112 single-strand repair genes [37]. Bottom: Heatmap representation of single-strand repair gene set expression in $\mathrm{d} 9$ and $\mathrm{d} 9$ cyt JAK2 $2 \mathrm{t}$ and $\mathrm{JAK} 2 \mathrm{~V} 617 \mathrm{~F}^{+} \mathrm{CD} 34^{+} \mathrm{P}$-ECs. Columns represent individual samples in experimental duplicates $(1,2)$. Two large differentially downregulated clusters of genes in $\mathrm{V} 617 \mathrm{~F}^{+}$samples are highlighted by yellow box with low variance between experimental duplicates (\#A) and higher variance (\#B). Blue box delimits cluster of genes upregulated with low variance between experimental duplicates in ${\mathrm{V} 617 \mathrm{~F}^{+}}$samples (\#C). See also Supplementary Fig. 2

(pATRT1989), showed low constitutive nuclear staining (Fig. 2a). These data suggested a mild degree of oxidative and replication stress, not converted into double-strand DNA breaks (absence of $\gamma \mathrm{H} 2 \mathrm{AX}$ foci) in PV and MF-1/2 (consistent with ongoing proliferation), and activated DDR signaling only at the post-PV MF-3 disease state.

Next, we determined oxidative damage and DDR activation in the differentiated JAK2wt and JAK2V617F iPSCs exposed to inflammatory cytokines. 8-oxoG staining was high in EBs derived from the JAK2wt iPSCs but undetectable in the $\mathrm{V} 617 \mathrm{~F}^{+}$counterparts (Fig. 2b). Assessment of DDR in vitro confirmed lower DNA damage (fewer $\gamma \mathrm{H} 2 \mathrm{AX}$ foci) in the d9 cyt $\mathrm{JAK}_{2} \mathrm{~V}_{617 \mathrm{~F}^{+}} \mathrm{CD} 4^{+} \mathrm{P}-$ ECs, compared with JAK2wt CD34 ${ }^{+}$P-ECs (Fig. 2c and Supplementary Fig. 2b), whereas inducing bystander DNA damage to the JAK2wt CD34 ${ }^{+}$P-ECs upon co-culture (Supplementary Fig. 2c). RAD51 recruitment to DNA damage sites, marked by $\gamma \mathrm{H} 2 \mathrm{AX}$ foci as readout for $\mathrm{HR}$ efficiency upon sequential staining [36], was not significantly altered (Fig. 2c and Supplementary Fig. 2b). With RAD51 accumulation following the trends of $\gamma \mathrm{H} 2 \mathrm{AX}$ levels these data suggest intact regulation of $\mathrm{HR}$ in the JAK2V617F ${ }^{+}$cells.

To examine whether observed protection against inflammation-evoked DNA damage in the JAK2V617F ${ }^{+}$cells is reflected in the global expression of DDR and DNA repair genes, we evaluated transcripts of 369 genes distributed to categories as described [37]. In individual heatmaps, clusters of upregulated genes in the $\mathrm{d} 9$ cyt JAK2wt CD34 ${ }^{+}$P-ECs were larger than in the JAK2V617F ${ }^{+} \mathrm{CD}^{+} 4^{+}$P-ECs (Fig. 2d and Supplementary Fig. 2d). DNA single-strand repair represented the most differentially expressed gene (DEG) set, as GSEA revealed enrichment for downregulated genes in the JAK2V617F ${ }^{+}$CD34 $^{+}$P-ECs (Fig. 2d).

We tested whether apparent DNA damage protection of $\mathrm{V} 617 \mathrm{~F}^{+}$cells is efficient only for inflammation-induced threshold dose of DNA damage, and thus represents adaptations to cell autonomous and microenvironmentdependent inflammatory stress in PV. Indeed, X-ray irradiation of the $\mathrm{d} 9 \mathrm{JAK} 2 \mathrm{~V}_{617 F^{+}} \mathrm{CD}^{+} 4^{+} \mathrm{P}$-ECs induced DDR signaling comparable or higher than in the JAK2wt cells (Supplementary Fig. 2e, f), suggesting that suprathreshold DNA damage activates robust DDR in JAK2V617F ${ }^{+}$cells.

\section{Inflammatory cytokines inhibit cell cycle of JAK2wt $\mathrm{CD}^{+} \mathrm{P}$-EC cells but fail to activate the p53/ p21waf1 checkpoint in the JAK2V617F ${ }^{+}$cells}

The observed absence of global DDR markers (including the lack of nuclear pATMS1981) in PV progenitors suggested unimpaired cell cycle progression of the JAK2V617F ${ }^{+}$cells rather than robust cell cycle interference, which would otherwise be expected to occur in an inflammatory environment [18, 38]. To analyze cell cycle progression of $\mathrm{d} 9$ and $\mathrm{d} 9$ cyt $\mathrm{JAK} 2 \mathrm{wt}$ or $\mathrm{JAK} 2 \mathrm{~V} 617 \mathrm{~F}^{+}$ $\mathrm{CD} 4^{+} \mathrm{P}-\mathrm{EC}$ cells, we synchronized the cells using growth factor withdrawal. To release from arrest, medium with growth factors and 5-bromo-2'-deoxyuridine (BrdU) was added and cell cycle progression monitored by flow cytometry (see Supplementary Fig. 3a for schematics of the experimental setup). The JAK2V617F ${ }^{+} \mathrm{CD} 34^{+}$P-EC cells revealed prolonged S-phase duration (Fig. 3a), consistent with an earlier study [39]. In contrast to the JAK2wt CD34 ${ }^{+}$ P-EC cells, the mutant $\mathrm{d} 9$ cyt cells lack activation of the p53/p21waf1 checkpoint (Fig. 3b, c). Low, but constitutive activation of Ser 317-phosphorylated Chk1 (pChk1S317), an ATR target site [40] is consistent with the observed constitutive pATRT1989 nuclear staining (Fig. 2a), marking proliferating cells enriched in $\mathrm{S}$ phase.

These data suggested that in the JAK2V617F ${ }^{+}$cells inflammatory factors induce only modest degree of DDR signaling, compatible with ongoing proliferation, thereby preventing (in the proliferative disease state) the buildup of more robust, ATM/p53/p21waf1-mediated signaling that could trigger cell cycle arrest and senescence.

\section{The JAK2V617F ${ }^{+}$CD34 $^{+}$P-ECs tightly regulate elevated ROS}

Inflammation associated with the $\mathrm{JAK}_{2} \mathrm{~V} 617 \mathrm{~F}^{+}$status was reported to increase ROS accumulation in the hematopoietic 


\section{a}

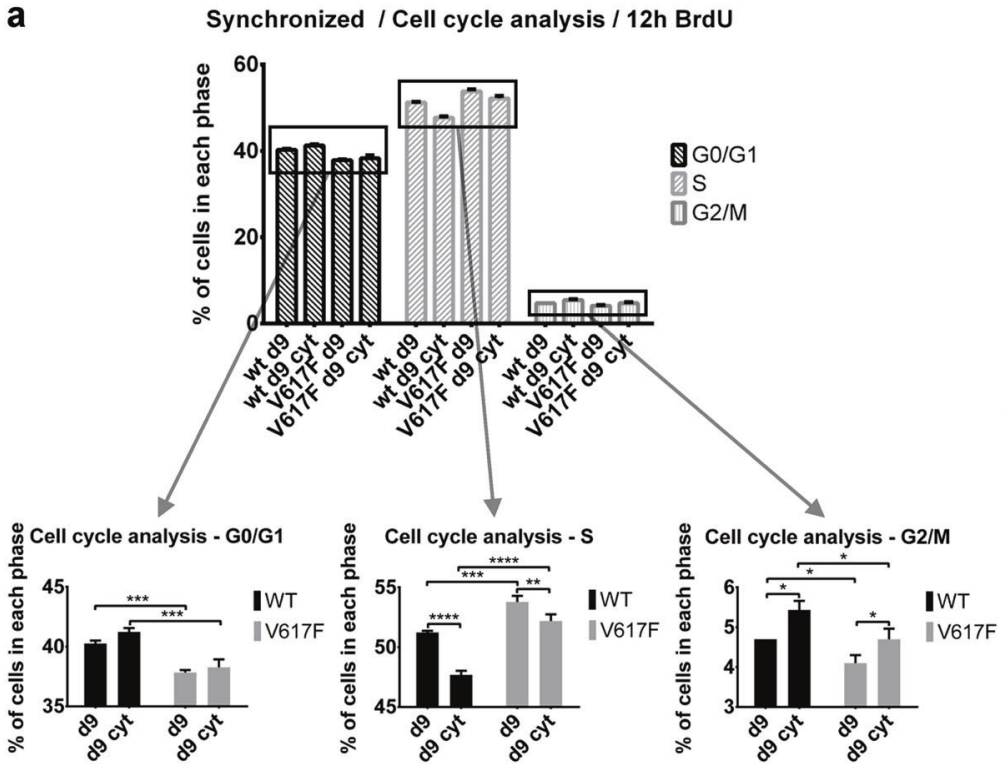

b

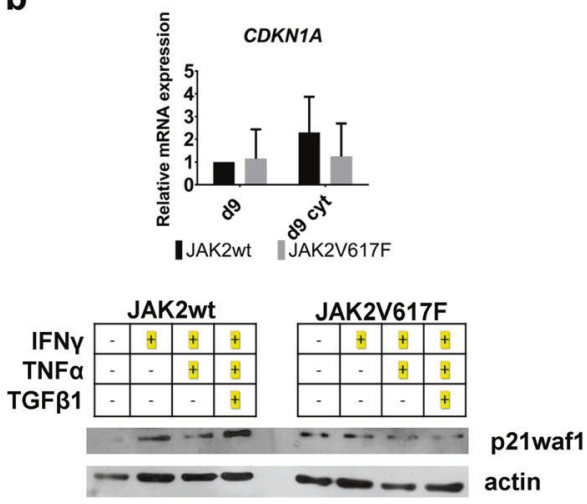

C

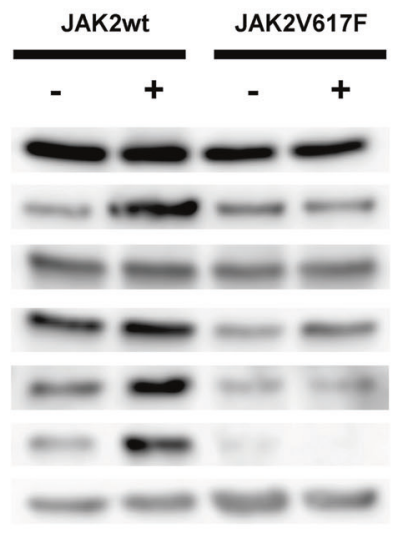

IFNy, TNF $\alpha$, TGF $\beta 1$

24h

Chk1

Chk1 S317

Chk2

Chk2 T68

p53

p53 S15

actin

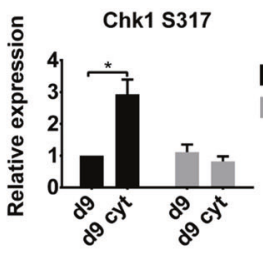

p53 S15

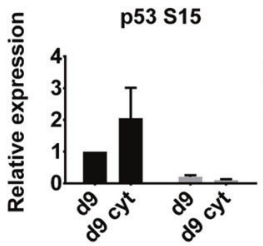

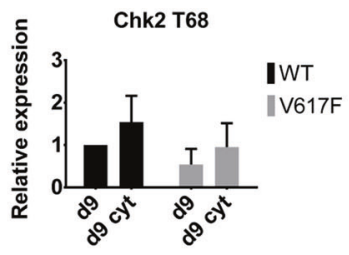

IWT

IV617F d

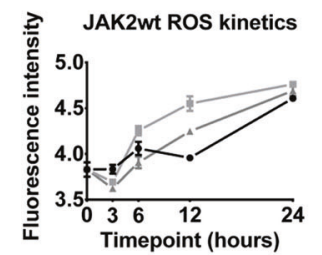

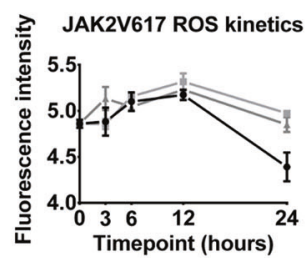

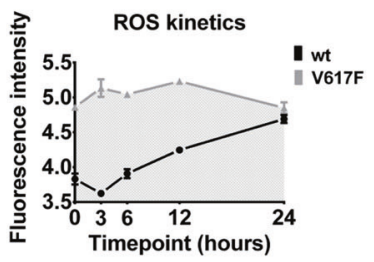

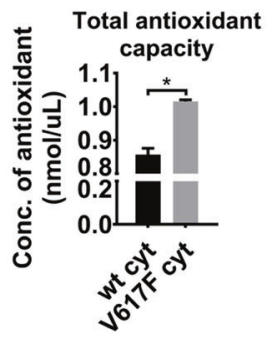

Fig. 3 Characterization of cell cycle progression, checkpoint activation and ROS production and buffering in JAK2 wt and JAK2V617F ${ }^{+}$CD34 + P-ECs. a Changes of cell cycle profiles of synchronized $\mathrm{CD}^{+} 4^{+}$P-EC cells untreated (d9) or treated with IFN $\gamma, \mathrm{TNF} \alpha$ and TGF $\beta 1$ for $24 \mathrm{~h}$ (d9 cyt). Bar charts show percentage of cell numbers \pm SD from three independent experiments in each phase of cell cycle. To further express the differences in each phase of cell cycle, the top of the chart bars were zoomed-in on separate charts. $* P \leq 0.05$, $* * P \leq 0.01$, $* * * P \leq 0.001, * * * * P \leq 0.0001$, two-way ANOVA. b Top: Quantitative RT-PCR analysis of CDKN1A (p21waf1) gene expression in day d9 or d9 cyt JAK2wt and JAK2V617F ${ }^{+} \mathrm{CD} 34^{+}$P-ECs. Values are shown as a mean \pm SD of three independent experiments. Bottom: Representative immunoblotting analysis with protein levels of p21waf1 in JAK2wt and $\mathrm{JAK}_{2} \mathrm{~V}_{17 \mathrm{~F}^{+}} \mathrm{CD} 4^{+} \mathrm{P}$-ECs upon treatment with IFN $\gamma$, TNF $\alpha$, and/or TGF $\beta 1$ in various combinations. $\mathbf{c}$ Western blotting analysis of total and phosphorylated forms of checkpoint proteins (Chk1, Chk2, and p53) in d9 and d9 cyt JAK2wt and JAK2V617F ${ }^{+}$CD34 ${ }^{+}$P-ECs. Charts show Chk1 S317, Chk2 T68, and p53 S15 relative expression (mean $\pm \mathrm{SD}, n$ $=2$ independent experiments) normalized to actin. $* P \leq 0.05$, two-way ANOVA. d Left: Kinetics of ROS production in three independent experiments presented as a mean \pm SD of samples' fluorescence intensities in JAK2wt and JAK2V617F ${ }^{+}$CD34 ${ }^{+}$P-ECs treated with defined combinations of inflammatory cytokines for $3,6,12$, and $24 \mathrm{~h}$ (left two charts); comparison of different kinetics of ROS production in JAK2wt and JAK2V617F ${ }^{+} \mathrm{CD} 34^{+} \mathrm{P}$-ECs after treatment with IFN $\gamma, \mathrm{TNF} \alpha$, and TGF $\beta 1$ for $24 \mathrm{~h}$ (right chart). Right: Total antioxidant capacity (concentration of antioxidant $\pm \mathrm{SD}, n=2$ independent experiments) in JAK2wt and JAK2V617F ${ }^{+} \mathrm{CD} 34^{+}$P-ECs treated with inflammatory cytokines for $24 \mathrm{~h}$. $* P \leq 0.05$, unpaired Student's $t$-test (two-tailed). See also Supplementary Fig. 3 
compartment [32]. We hypothesized that the JAK2V617F $\mathrm{F}^{+}$ progenitors must actively upregulate antioxidant systems to avoid detrimental effects of oxidative stress, even more than in other inflammation-associated cancers [41]. Kinetic measurements of ROS levels in the d9 cyt JAK2wt and $\mathrm{JAK} \mathrm{V} 617 \mathrm{~F}^{+} \mathrm{CD}^{+} 4^{+} \mathrm{P}$-ECs revealed that while ROS levels increase over time of cytokine exposure in the JAK2wt cells, the JAK2V617F ${ }^{+}$cells maintained a relatively high yet stable ROS levels (Fig. 3d). This was associated with a significantly increased antioxidant capacity of the JAK2V617F ${ }^{+} \mathrm{CD} 34^{+} \mathrm{P}$-ECs when compared with the JAK2wt controls (Fig. 3d). Accordingly, we observed a differentially upregulated expression of peroxiredoxins, cyclooxygenases, thioredoxins, and catalase in the JAK2V617 ${ }^{+}$CD34 ${ }^{+}$P-ECs. Further analysis of antioxidant defense enzymes revealed several enzyme activities upregulated in the JAK2V617F ${ }^{+} \mathrm{CD} 34^{+}$P-ECs (Supplementary Fig. 3b).

These results suggested relatively increased but tightly controlled ROS levels in the JAK2 $2617 \mathrm{~F}^{+}$compartment.

\section{DUSP1 protects the JAK2V617F ${ }^{+}$progenitors against stress response signaling and DDR}

We hypothesized that the observed intrinsic resistance of the JAK2 $2617 \mathrm{~F}^{+}$progenitors against inflammation-evoked DNA damage involves stress-activated protein kinases (SAPKs) from mitogen-activated protein kinase (MAPK) superfamily [42]. SAPKs include Jun kinases (JNKs) and p38MAPK that maintain survival of cells exposed to environmental stresses including inflammation [43, 44], however, when p38MAPK activation is too strong, cells including the JAK2 $2617 \mathrm{~F}^{+}$progenitors, undergo apoptosis or senescence $[45,46]$. Dual-specificity MAPK phosphatases (DUSPs) support cancer cell survival by buffering SAPKs activities under inflammatory conditions [47]. Some of the DUSPs, including DUSP1, were previously implicated in cellular protection against accumulation of DNA damage and DDR checkpoints [48]. Analysis of DUSP gene family revealed mostly upregulated genes in the JAK2V617F ${ }^{+} \mathrm{CD}^{2} 4^{+}$P-ECs, either intrinsically with further increase in the d9 cyt cultures, or differentially upregulated in the d9 cyt $\mathrm{JAK}^{2} \mathrm{~V} 617 \mathrm{~F}^{+}$cells when compared with the JAK2wt and untreated mutant CD34 ${ }^{+}$P-ECs (Fig. 4a). Among the intrinsically upregulated DUSPs in JAK2V617F ${ }^{+}$CD34 $^{+}$P-ECs dominated those with substrate specificity for JNK and p38MAPK: DUSP1, DUSP16, and DUSP8 [47]. Recently, JAK2V617F was shown to induce expression of DUSP1 in a cellular model [49]. In a mouse model of c-Jun-mediated fibrosis, Dusp1 overexpression represented the main negative feedback loop that antagonized p38MAPK/JNK activities [50]. Moreover, DUSP1, a known NF- $\mathrm{KB}$ target [51], clustered to differentially overexpressed genes in our NF- $\mathrm{BB}$ gene set characterizing the network upregulated in the JAK2V617F $\mathrm{CD} 34^{+}$P-ECs (Supplementary Fig. 1k). The DUSP1 protein was highly inducible in the $\mathrm{d} 9$ cyt $\mathrm{JAK} \mathrm{V} 617 \mathrm{~F}^{+}$ CD $34^{+}$P-ECs (Fig. 4b). Our analyses of clinical patients' $\mathrm{BM}$ biopsies along PV progression revealed moderate DUSP1 positivity in all samples at all PV disease stages (Fig. 4c). DUSP6 was expressed to a much lesser degree in patients' BM (Supplementary Fig. 4a). Together, these findings indicated a role of DUSP1 in limiting the stressinduced signaling in PV progenitors, thereby preventing a robust activation of cell cycle checkpoints and cellular senescence.

To functionally assess the contribution of high DUSP1 expression in the $\mathrm{JAK} 2 \mathrm{~V} 617 \mathrm{~F}^{+}$progenitors, we used human erythroleukemia (HEL) cells harboring the V617F oncogenic mutation as well as CRISPR/Cas9 JAK2-edited (JAK2wt) HEL cells (see Supplementary Materials and Methods for details on JAK2-edited HEL cells) and exposed them to a DUSP1/6 inhibitor ((E)-2-benzylidene-3(cyclohexylamino)-2,3-dihydro-1H-inden-1-one; BCI) [52]. Based on preliminary testing (Supplementary Fig. 4b), we chose 1-h BCI treatment to examine the effect of DUSP1/6 inhibition on cell signaling and the fate of unstressed and inflammatory factor-stressed JAK2V617F and JAK2-edited (JAK2wt) HEL cells. We observed more elevated DUSP1 and DUSP6 in the HELV617F cells compared with the HEL-edited JAK2wt cells, however, whereas DUSP6 expression after cytokine treatment sharply decreased, the post-treatment DUSP1 levels increased (Supplementary Fig. 4c). These results suggested that DUSP1 is the main target of the BCI inhibitor in these cells.

As expected, Thr180/Tyr182-phosphorylated p38MAPK (p38 T180/Y182) and Thr183/Tyr185-phosphorylated JNK (JNK T183/Y185) increased after BCI treatment in both HELV617F and HEL-edited cells, regardless of cytokine treatment (Fig. 4d). However, a major difference was observed in cytokine-untreated HEL cells, as $\gamma \mathrm{H} 2 \mathrm{AX}$ was largely unaffected in BCI-treated HEL-edited cells, contrary to enhanced $\gamma \mathrm{H} 2 \mathrm{AX}$ in the HELV617F cells (Fig. 4d). Nonresponsiveness of cytokine-untreated HEL-edited cells to BCI was corroborated by analyses of cell cycle progression and apoptosis. Whereas the increased SAPKs activities after BCI exposure corresponded to significantly more cells in G1 phase and increased apoptosis of the HELV617F cells, the proportions of G1 and apoptotic HEL-edited cells remained unchanged (Fig. 4d). The HEL-edited cells accumulated DNA damage $(\gamma \mathrm{H} 2 \mathrm{AX})$ and increased apoptotic cells only when the BCI exposure was combined with cytokine treatment (Fig. 4d). To confirm that the BCI-inhibitory effects are attributable to DUSP1 inhibition, we knocked down DUSP1 by small interfering RNA (siRNA) in HELV617F and HEL-edited cells (Supplementary Fig. 4d). Indeed, siRNA-mediated 
a

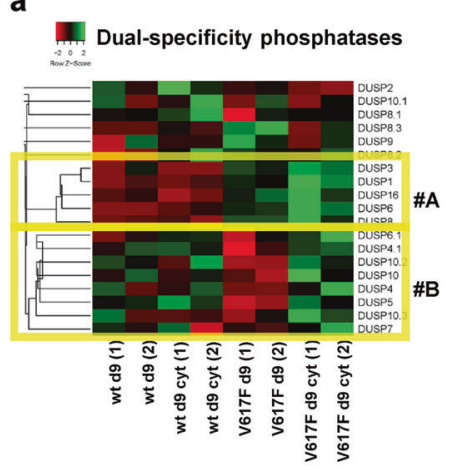

d

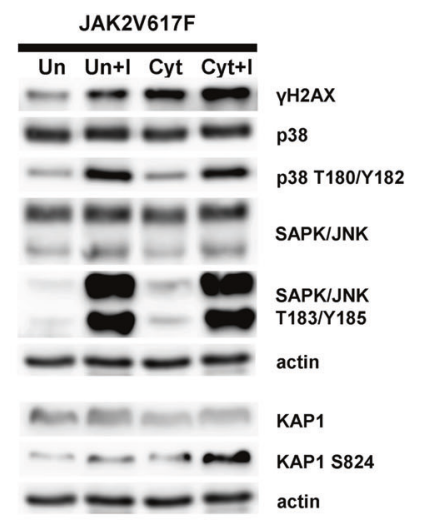

b

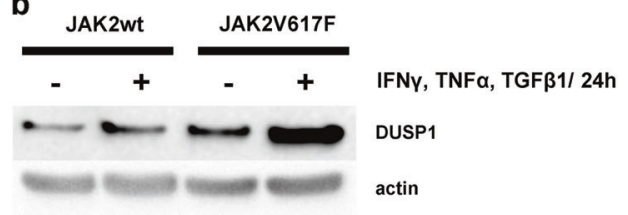

C

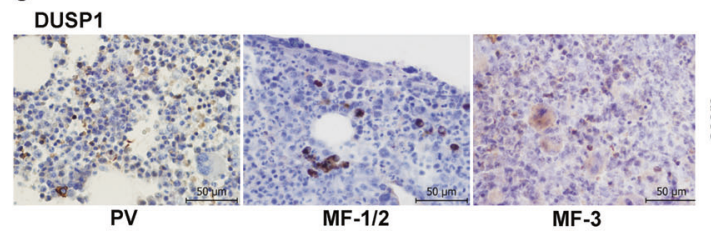

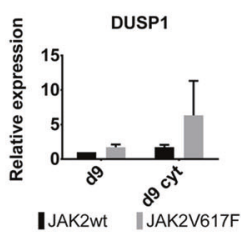

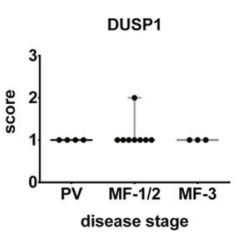

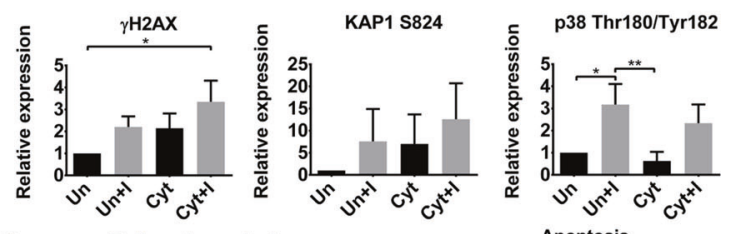
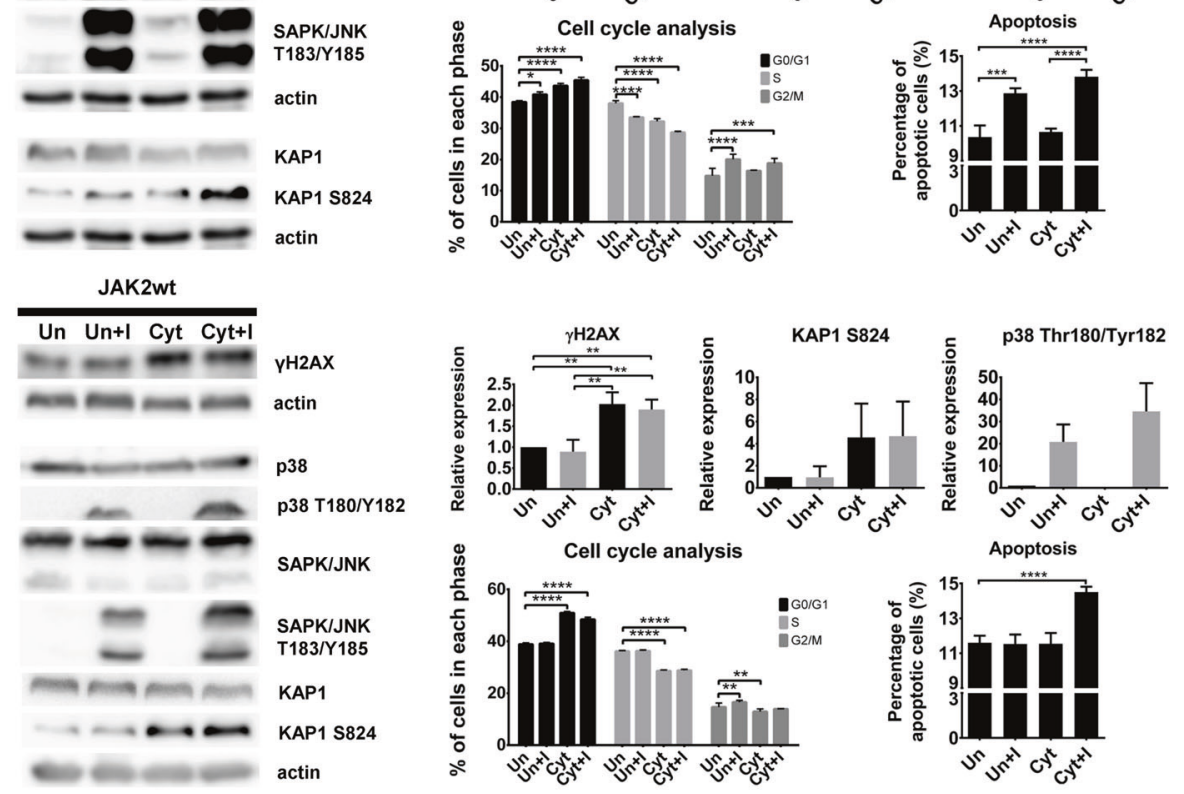

e
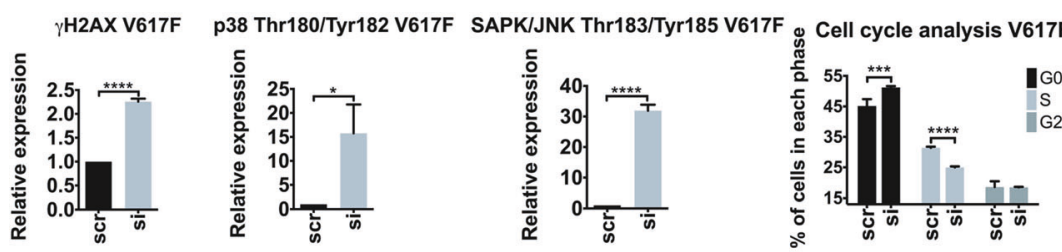

Apoptosis V617F
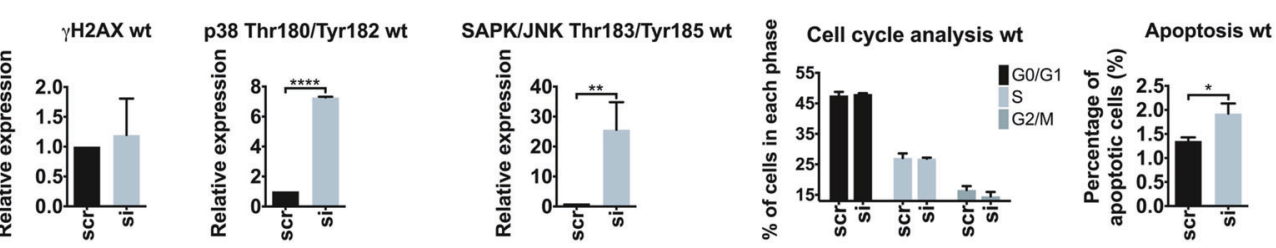

depletion of DUSP1 induced changes in protein expression and cell fate phenotypes similar to BCI treatment (Fig. 4e and Supplementary Fig. 4e).
Collectively, all these data indicate that the JAK2V617F $\mathrm{F}^{+}$ progenitors are strongly dependent on DUSP1 activity for proliferation and survival and that high DUSP1 expression 
Fig. 4 JAK2V617F cell-specific DUSP1 overexpression and DUSP1dependent proliferation and survival of JAK2V617F cells. a Cluster analysis of heatmap representation of differential expression of DUSP genes in $\mathrm{d} 9$ and $\mathrm{d} 9$ cyt JAK2wt and JAK2V617F ${ }^{+} \mathrm{CD} 34^{+}$P-ECs. Columns in the heatmap represent individual samples in experimental duplicates $(1,2)$. Heatmap was constructed by using Kendall's Tau Distance Measurement Method and Centroid Linkage Clustering Method. Cluster \#A contains intrinsically upregulated DUSPs in d9 and cluster \#B contains DUSPs upregulated in $\mathrm{d} 9$ cyt $\mathrm{JAK} 2 \mathrm{~V} 617 \mathrm{~F}^{+}$ CD34 ${ }^{+}$P-ECs. b Left: Western blotting analysis of DUSP1 in untreated (d9) or treated with IFN $\gamma, \mathrm{TNF} \alpha$ and TGF $\beta 1$ for $24 \mathrm{~h}$ (d9 cyt) JAK2wt and JAK2V617F ${ }^{+}$CD34 ${ }^{+}$P-ECs. Right: This chart shows relative expression of DUSP1 in three independent experiments normalized to actin. c Representative IHC staining of BM sections for DUSP1 among different disease stages of PV progression. Scale bar, $50 \mu \mathrm{m}$. Boxplot shows quantification of the number of cells expressing DUSP1 in sections of patients from grouped disease stages. $\mathbf{d}$ Western blotting of indicated markers of DDR $(\gamma \mathrm{H} 2 \mathrm{AX}, \mathrm{KAP} 1)$ and SAPK (p38MAPK, JNK) activation, changes in cell cycle profile and percentage of apoptotic cells for HELV617F cells (JAK2V617F; upper panels) and HEL-edited cells (JAK2wt; bottom panels) untreated (Un) or treated with inflammatory cytokines (Cyt) and without or with exposure to BCI $(\mathrm{Un}+\mathrm{I}$ or Cyt $+\mathrm{I})$. Charts show $\gamma \mathrm{H} 2 \mathrm{AX}$, Ser 824phosphorylated KAP1 (KAP1 S824) and p38 T180/Y182 relative expression in three independent experiments normalized to actin. KAP1 S824 is an ATM target site [82]. Cell cycle distribution and apoptosis bar charts show percentage of cell numbers \pm SD of four independent experiments in each phase of cell cycle and percentage of apoptotic cells, respectively. $* P \leq 0.05, * * P \leq 0.01$, *** $P \leq 0.001$, $* * * * P \leq 0.0001$, one-way (western blotting) and two-way (cell cycle and apoptosis) ANOVA. e Effect of DUSP1 knock-down in three independent experiments in cytokine-untreated HELV617F and HELedited cells on $\gamma \mathrm{H} 2 \mathrm{AX}$ and SAPK (p38MAPK, JNK) expression (left graphs), and cell cycle distribution and apoptosis (right graphs). Effects of DUSP1-specific siRNA (si) are compared with scrambled RNA control (scr). Data are normalized and plotted as in d. $* P \leq 0.05$, $* * P \leq 0.01, * * * P \leq 0.001, * * * * P \leq 0.0001$, two-way ANOVA (cell cycle) and unpaired Student's $t$-test (two-tailed, the rest). See also Supplementary Fig. 4

provides adaptation of the $\mathrm{JAK} 2 \mathrm{~V} 617 \mathrm{~F}^{+}$progenitors to inflammatory stress.

\section{Discussion}

This study contributes to understanding of cancer in general, and pathobiology of PV in particular, at several levels: (i) conceptual; (ii) mechanistic; (iii) clinically related, with therapeutic implications.

From a broader conceptual perspective, our results highlight intriguing differences between the impact of oncogenes activated in common human malignancies, and the JAK2V617F oncogene driving the MPNs including PV. Thus, the former oncogenes, such as Ras, Myc, etc. cause endogenous DNA damage at early stages of tumorigenesis, triggering cell cycle checkpoints and the ensuing cellular senescence or cell death, events that originally led us and others to formulate the concept of DDR as an intrinsic barrier against activated oncogenes and tumor progression of major types of carcinomas [53-55]. This concept, which we have later extended also to brain tumors [56, 57] and $M L L-E N L$ oncogene-induced leukemogenesis [58], furthermore explains the disease-evolutionary pressure to select for inactivating mutations in checkpoint genes such as TP53, ATM or CHK2 [53-55, 59], as secondary events that allow checkpoint bypass and tumor cell proliferation, at the expense of high genomic instability. In contrast, we report here that such fate is largely prevented in the JAK2V617F-driven PV progenitor cells known to show long-term cell proliferation with a rather stable genome [8, 60]. Our data suggest that this unorthodox fate of PV cells reflects the lack of any robust endogenous DNA damage or checkpoint activation, despite the complex PV-associated inflammatory and oxidative stresses that are known to trigger pronounced DDRs and cellular senescence or death in other inflammation-associated settings [18, 38, 61]. As explained below, we propose that the underlying mechanisms for such unique pathobiological behavior reflect features "in-built" among the effects of the JAK2V617F oncogene, effects that mitigate the overall extent and/or impact of the inflammatory and oxidative insults in PV progenitors, thereby allowing for chronic proliferation in the indolent phase of JAK2V617F ${ }^{+}$MPNs [8, 60].

Some studies including in vitro model experiments and cultured patients' cells, suggested increased DNA damage in the JAK2V617F hematopoietic compartment [32, 33, 62], a result at odds with our present dataset. However, if the PV JAK2V617F cells indeed accumulated high ROS-dependent oxidative stress [32] or featured enhanced DNA breakage [33], the proliferative stage of the disease would be shorter and compromised by checkpoint activation, events that do not match the known disease course or our results. Here, we identified some mechanistic aspects downstream of JAK2V617F that explain the apparent discrepancies and the unique behavior of the PV progenitors. First, using complementary models and approaches, we identified DUSP1 phosphatase as a candidate factor that mediates signaling adaptation to inflammatory stress in JAK2V617F ${ }^{+} \mathrm{PV}$ progenitors. DUSP1 counteracts JNK and p38MAPK activities [47], the key kinases activated in stressed hematopoiesis, including inflammation-exposed HSCs [7, 44]. We found DUSP1 upregulated downstream of the JAK2V617F-

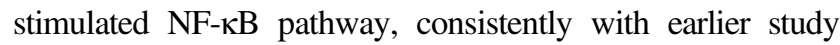
[51]. Notably, we report that DUSP1 is highly expressed specifically in $\mathrm{JAK}_{2}$ V617F ${ }^{+} \mathrm{CD}^{+} 4^{+} \mathrm{P}-\mathrm{EC}$, in HELV617F cells and in the PV patients' BM. Furthermore, our experimental chemical inhibition as well as siRNA-mediated knockdown of DUSP1 leads to JNK/p38MAPK reactivation, accumulation of DNA damage $(\gamma \mathrm{H} 2 \mathrm{AX})$ and accelerated cell cycle arrest and apoptosis of $\mathrm{JAK} 2 \mathrm{~V} 617 \mathrm{~F}^{+}$, but not JAK2wt cells. These data indicate that $\mathrm{JAK} 2 \mathrm{~V} 617 \mathrm{~F}^{+}$progenitors depend on DUSP1 activity for proliferation and survival, and that elevated DUSP1 provides adaptation of JAK2V617F 
progenitors to inflammatory stress. Another important aspect of our data in this context is the evidence for a hierarchical cytokine-induced inflammatory network in JAK2V617F ${ }^{+}$ $\mathrm{CD}_{3}{ }^{+} \mathrm{P}-\mathrm{EC}$, including intrinsic IFN $\gamma$ priming fueled by JAK2V617F. As a consequence of the specific JAK2V617Fmediated inflammatory phenotype with ensuing potentially genotoxic ROS, we identified a hyper-activated "adaptive" ROS-buffering system. Given that DUSP activity is sensitive to ROS-mediated oxidation [63], our results suggest that upregulation of the antioxidant defense driven by JAK2V617F is required for enhanced DUSP1 activity. We propose that cooperation between high antioxidant buffering capacity and DUSP1 activity form a regulatory loop that allows proliferation and survival of $\mathrm{JAK} 2 \mathrm{~V} 617 \mathrm{~F}^{+}$progenitors by protecting them against endogenous DNA damage. Another complementary factor that can help avoid extensive DNA damage is RECQL5, a JAK2V617F/STAT-upregulated helicase that mitigates replication stress and thereby helps maintain genomic stability in the chronic phase of MPN [34].

Last but not least, in the clinical context, our dataset has implications for understanding the disease course and potentially innovative treatment. Thus, our model experiments are consistent with our biomarker analyses of patients' BM biopsies from diverse stages of PV. Our mechanistic data also suggest that with increasing risk of fibrotic transformation, associated with enhanced inflammation and ROS production [4, 64], the "protective" DUSP1 activity gets gradually suppressed, resulting in sustained JNK/p38MAPK activation and ensuing expression of inflammatory mediators, promoting fibrotic transformation [65], dysmegakaryopoiesis [10], and anemia associated with MF [66]. In summary, our results suggest that DUSP1 addiction in the early stages of PV offers not only insights into the unique pathobiology, but also a potential therapeutic target, as inhibition of DUSP1 leading to JNK/p38MAPK hyperactivation may provide a strategy for elimination of the cycling JAK2V617F ${ }^{+}$progenitors. Given that JAK2V617F ${ }^{+}$ progenitors maintain a relatively high ROS status, synthetic lethality-based approach can be exploited to selectively target these cells by escalating the oxidative stress using MTH1 inhibitors [67] and simultaneously target DUSP1 for DNA damage elevation to the level inducing cell death.

\section{Materials and methods}

\section{Culture of undifferentiated iPSCs}

Patient-specific iPSC line with JAK2V617F genotype (PVB1.4, obtained from original investigators J.T. Prchal and L. Cheng) was described in detail previously [12, 68]. Control iPSC line with wild-type JAK2 genotype
(BXS0116) was obtained from ATCC and its characterization including capacity to activate DDR was published elsewhere [13]. iPSCs were cultured as described [12]. For radiation experiments, cells were irradiated with $2 \mathrm{~Gy}$ of $\mathrm{X}$ rays (RS Research Cabinet, Xstrahl, Surrey, UK) and incubated $3 \mathrm{~h}$ before harvesting.

\section{iPSC hematopoietic differentiation}

$\mathrm{CD} 34^{+} \mathrm{P}$-ECs with hematopoietic differentiation program were generated from iPSCs using published protocol [14]. For cytokines used and workflow of iPSCs differentiation see Supplementary Materials and Methods. To test cytokine effects, IFN $\gamma(100 \mathrm{U} / \mathrm{ml}), \mathrm{TNF} \alpha(50 \mathrm{ng} / \mathrm{ml})$, and TGF $\beta 1(5 \mathrm{ng} / \mathrm{ml})$ were added to culture media $24 \mathrm{~h}$ prior to harvesting cells for analysis (day 9 of differentiation; all cytokines purchased from ProSpec-Tany TechnoGene, Rehovot, Israel).

\section{Generation of JAK2-edited (JAK2wt) HEL cell line}

JAK2V617F mutation in HEL cells (obtained from DSMZ - German Collection of Microorganisms and Cell Cultures) was repaired by HR using CRISPR/Cas9 (CRISPR construct pXPR001; Addgene \#49535) with inserted sgRNA (5'- ACGAGAGTAAGTAAAACTAC-3') and long dsDNA template spanning JAK2 exon 24 (1818 bp). HEL cells were electroporated using Amaxa ${ }^{\mathrm{TM}}$ Nucleofector ${ }^{\mathrm{TM}}$ (kit V, program X-005), single-cell sorted $48 \mathrm{~h}$ post electroporation based on their fluorescence status and single-cell clones expanded. Functional comparison of HEL-edited (JAK2wt) with control parental (unedited HELV617F) clones is described in Supplementary Materials and Methods.

\section{Microarray and data analysis}

Two biological replicates per group were used. Total RNA was isolated using TRI Reagent (Sigma-Aldrich) and RNA integrity tested by Agilent 2100 Bioanalyzer (RNA integrity number > 9; Agilent Technologies). RNA was amplified and labeled using Illumina TotalPrep RNA Amplification Kit (Ambion; Thermo Fisher) and hybridized on HumanHT-12 v4 Expression BeadChips (Illumina). Raw data were processed using Genome Studio software (version 1.9.0.24624; Illumina) and analyzed within the oligo and limma packages of the Bioconductor [69-71]. Moderated $t$-test was used to detect DEGs between the sample groups: at least twofold change in gene expression and Storey's $q$-value $<0.1$ were required [72]. MIAME compliant data were deposited to the Array Express database (E-MTAB-7693). Heatmapper tool was used to generate heatmaps to visualize differences in gene expression [73]. Heatmaps were constructed with Pearson 
Distance Measurement Method and Average or Centroid Linkage Clustering Method.

\section{Pathway over-representation analysis and GSEA}

Over-representation analysis of DEGs and generation of interaction network module maps using gene regulatory interactions was carried out with the ConsensusPathDB software (Release 32 (11.01.2017)) using 12 biological pathways databases (Wikipathways, Inoh, Netpath, Smpdb, Pharmgkb, Biocarta, Kegg, Reactome, Humancyc, Signalink, Ehmn, and Pid) to identify pathways associated with the mRNAs differentially expressed in studied samples [74]. GSEAs were performed with GSEA v3.0 software [75]. Ratio_of_Classes was used as a metric to rank genes.

\section{Quantitative real-time polymerase chain reaction}

Total RNA was reverse transcribed (Roche) and treated with TURBO DNA-free DNase (Thermo Fisher). LightCycler 480 platform (Roche) was employed as described [76], using $R P L P O$ as a reference gene. Primers are listed in Supplementary Table 1 .

\section{Flow cytometry analysis}

Flow cytometry analysis was done using Cytomics FC500 and CXP software (Beckman Coulter). For cell cycle distribution analysis and apoptosis, CD34 ${ }^{+}$P-ECs were treated as described in Supplementary Fig. 3a and then labeled with $10 \mu \mathrm{M}$ BrdU (Sigma-Aldrich). Combined BrdU and propidium iodide staining was as described [77], gating strategy is shown in Supplementary Materials and Methods. ROS production was measured by CellROX Green Flow Cytometry Assay Kit in CD $34^{+}$P-ECs treated with defined combinations of cytokines for 3, 6, 12, and $24 \mathrm{~h}$. The surface markers CD34, CD41, and CD43 were detected with the respective antibodies using dilutions specified in Supplementary Table 2. In all experiments, appropriate isotype control antibodies were used.

\section{Enzyme assay}

The activity of antioxidative enzymes was determined according to standard methods [78, 79]. The enzyme activity was expressed in units per gram of total protein. The total antioxidant capacity was determined using the Total Antioxidant Capacity Assay Kit (Sigma-Aldrich).

\section{Immunocytochemistry on EBs in agarose gel matrix saturated with paraffin}

Paraffin-embedded JAK2wt and JAK2V617F ${ }^{+}$EBs were prepared as described [80]. Nonspecific binding sites were blocked with $0.2 \%$ bovine serum albumin for $1 \mathrm{~h}$, sections stained with primary antibody at RT for $1 \mathrm{~h}$. EnVisionTM system (Dako, Glostrup, Denmark) including horseradish peroxidase and diaminobenzidine $\left(\mathrm{DAB}^{+}\right.$, Dako) was used for antibody detection; cell nuclei were counterstained by hematoxylin. Images were acquired using an Olympus BX51 inverted microscope equipped with ColorViewIII digital CCD camera.

\section{Immunocytochemistry of CD34 ${ }^{+}$P-ECs}

Single-cell suspension from CD $34^{+}$P-EC was cytospined onto microscope slides, cells fixed by $3 \%$ formaldehyde, permeabilized and stained with respective antibodies listed in Supplementary Table 2.

\section{Inhibition of DUSPs}

To test effects of DUSP1/6 inhibitor (BCI-CAS 15982-840 , Merck), untreated and cytokine-treated HEL cells were incubated with $10 \mu \mathrm{M} \mathrm{BCI}$ (dissolved in DMSO) for defined times $(0.5,1,2,4$, and $6 \mathrm{~h})$, in case of cytokine-treated cells prior the end of 24-h cytokine treatment.

\section{DUSP1 silencing by siRNA}

Control parental HEL cells and HEL-edited cells were transfected with validated Silencer Select siRNA (Thermo Fisher Scientific, Assay ID: s4363) and with corresponding recommended Silencer Select siRNA-negative control (scr) using RNAiMAX transfection reagent (Thermo Fisher Scientific). Transfected cells were subsequently harvested at 24,48 , and $72 \mathrm{~h}$ after transfection for RNA and $72 \mathrm{~h}$ for protein lysates.

\section{IHC of patients' samples}

Formalin-fixed, paraffin-embedded tissue samples were sectioned and processed using standard protocols for biopsy processing. BM trephine biopsies were demineralized by $10 \%$ chelatone $3(\mathrm{pH} 8)$ directly after fixation. To grade fibrosis, Gomori's silver impregnation for reticulin was performed. Visualization of antigens is described in Supplementary Materials and Methods, with antibodies specified in Supplementary Table 2. For staining evaluation, published scoring was chosen [81], and the results presented as boxplots where each dot represents a patient's sample plotted against the staining score. The whiskers indicate samples within the minimum and maximum values and the line across the box represents median. More details are in Supplementary Materials and Methods. 


\section{Patients' samples}

Human BM samples were obtained from the Department of Clinical and Molecular Pathology, University Hospital Olomouc (UHOL), Olomouc, Czech Republic. The original examinations were obtained with the approval of the IRB committee of the UHOL and according to the Declaration of Helsinki. Additional information on patient samples is available in Supplementary Table 3.

\section{Statistical analysis}

Mean values \pm SD are shown. Mann-Whitney test, Student's unpaired two-tailed $t$-test, two-way and one-way ANOVA with Bonferroni correction for multiple comparisons were used for obtaining statistical significance values (using Graph-Pad Prism Version 6.0).

Acknowledgements The authors thank Dr. J.T. Prchal, University of Utah and Dr. L. Cheng, JHU, Baltimore, for providing iPSCs and O. Babosova, J. Stastna, I. Stetkova, and L. Voznakova for technical assistance.

Funding Czech Science Foundation Project 14-10687P (PV), LTAUSA17142 project (JS, LL, and VD), Internal Grant Agency of Palacky University, Project IGA_LF_2018_010 (JS and PK), Danish Council for Independent Research (JB), Swedish Research Council (JB), and European Regional Development Fund-Project ENOCH/ CZ.02.1.01/0.0/0.0/16_019/0000868 (ZH and JB).

\section{Compliance with ethical standards}

Conflict of interest The authors declare that they have no conflict of interest.

Publisher's note: Springer Nature remains neutral with regard to jurisdictional claims in published maps and institutional affiliations.

Open Access This article is licensed under a Creative Commons Attribution 4.0 International License, which permits use, sharing, adaptation, distribution and reproduction in any medium or format, as long as you give appropriate credit to the original author(s) and the source, provide a link to the Creative Commons license, and indicate if changes were made. The images or other third party material in this article are included in the article's Creative Commons license, unless indicated otherwise in a credit line to the material. If material is not included in the article's Creative Commons license and your intended use is not permitted by statutory regulation or exceeds the permitted use, you will need to obtain permission directly from the copyright holder. To view a copy of this license, visit http://creativecommons. org/licenses/by/4.0/.

\section{References}

1. Zambetti NA, Ping Z, Chen S, Kenswil KJG, Mylona MA, Sanders MA, et al. Mesenchymal inflammation drives genotoxic stress in hematopoietic stem cells and predicts disease evolution in human pre-leukemia. Cell Stem Cell. 2016;19:613-27.
2. Campbell PJ, Green AR. The myeloproliferative disorders. N Engl J Med. 2006;355:2452-66.

3. Vainchenker W, Kralovics R. Genetic basis and molecular pathophysiology of classical myeloproliferative neoplasms. Blood. 2017;129:667-79.

4. Hasselbalch HC, Bjørn RnME. MPNs as inflammatory diseases: the evidence, consequences, and perspectives. Mediators Inflamm. 2015;2015:e102476

5. Vaidya R, Gangat N, Jimma T, Finke CM, Lasho TL, Pardanani A, et al. Plasma cytokines in polycythemia vera: phenotypic correlates, prognostic relevance, and comparison with myelofibrosis. Am J Hematol. 2012;87:1003-5.

6. Schepers K, Pietras EM, Reynaud D, Flach J, Binnewies M, Garg $\mathrm{T}$, et al. Myeloproliferative neoplasia remodels the endosteal bone marrow niche into a self-reinforcing leukemic niche. Cell Stem Cell. 2013;13:285-99.

7. Pietras EM. Inflammation: a key regulator of hematopoietic stem cell fate in health and disease. Blood. 2017;130:1693-8.

8. Tefferi A, Guglielmelli P, Larson DR, Finke C, Wassie EA, Pieri $\mathrm{L}$, et al. Long-term survival and blast transformation in molecularly annotated essential thrombocythemia, polycythemia vera, and myelofibrosis. Blood. 2014;124:2507-13.

9. Besancenot R, Chaligné R, Tonetti C, Pasquier F, Marty C, Lécluse $\mathrm{Y}$, et al. A senescence-like cell-cycle arrest occurs during megakaryocytic maturation: implications for physiological and pathological megakaryocytic proliferation. PLoS Biol. 2010. https://doi.org/10.1371/journal.pbio.1000476.

10. Desterke C, Bilhou-Nabéra C, Guerton B, Martinaud C, Tonetti C, Clay D, et al. FLT3-mediated p38-MAPK activation participates in the control of megakaryopoiesis in primary myelofibrosis. Cancer Res. 2011;71:2901-15.

11. Walter D, Lier A, Geiselhart A, Thalheimer FB, Huntscha S, Sobotta MC, et al. Exit from dormancy provokes DNA-damageinduced attrition in haematopoietic stem cells. Nature. 2015;520:549-52.

12. Ye Z, Liu CF, Lanikova L, Dowey SN, He C, Huang X, et al. Differential sensitivity to JAK inhibitory drugs by isogenic human erythroblasts and hematopoietic progenitors generated from patient-specific induced pluripotent stem cells. Stem Cells Dayt Ohio. 2014;32:269-78.

13. Mills RJ, Titmarsh DM, Koenig X, Parker BL, Ryall JG, QuaifeRyan GA, et al. Functional screening in human cardiac organoids reveals a metabolic mechanism for cardiomyocyte cell cycle arrest. Proc Natl Acad Sci USA. 2017;114:E8372-81.

14. Kennedy M, Awong G, Sturgeon CM, Ditadi A, LaMotte-Mohs $\mathrm{R}$, Zúñiga-Pflücker JC, et al. T lymphocyte potential marks the emergence of definitive hematopoietic progenitors in human pluripotent stem cell differentiation cultures. Cell Rep. 2012;2:1722-35.

15. Chen E, Beer PA, Godfrey AL, Ortmann CA, Li J, Costa-Pereira $\mathrm{AP}$, et al. Distinct clinical phenotypes associated with JAK2V617F reflect differential STAT1 signaling. Cancer Cell. 2010;18:524-35.

16. Fleischman AG, Aichberger KJ, Luty SB, Bumm TG, Petersen $\mathrm{CL}$, Doratotaj $\mathrm{S}$, et al. TNF $\alpha$ facilitates clonal expansion of JAK2V617F positive cells in myeloproliferative neoplasms. Blood. 2011;118:6392-8.

17. Chagraoui H, Komura E, Tulliez M, Giraudier S, Vainchenker W, Wendling F. Prominent role of TGF-beta 1 in thrombopoietininduced myelofibrosis in mice. Blood. 2002;100:3495-503.

18. Hubackova S, Kucerova A, Michlits G, Kyjacova L, Reinis M, Korolov O, et al. IFN $\gamma$ induces oxidative stress, DNA damage and tumor cell senescence via TGF $/$ SMAD signaling-dependent induction of Nox4 and suppression of ANT2. Oncogene. 2016;35:1236-49. 
19. Wu X, Dao Thi VL, Huang Y, Billerbeck E, Saha D, Hoffmann $\mathrm{H}-\mathrm{H}$, et al. Intrinsic Immunity Shapes Viral Resistance of Stem Cells. Cell. 2018;172:423-38.

20. Haas S, Hirche C, Schnell A, Sönmezer C, Langstein J, Wurzer S, et al. A stem cell-based epigenetic memory mediates interferon response-heterogeneity within the hematopoietic system. Blood. 2017;130(Suppl1):634.

21. Kleppe M, Spitzer MH, Li S, Hill CE, Dong L, Papalexi E, et al. Jak1 integrates cytokine sensing to regulate hematopoietic stem cell function and stress hematopoiesis. Cell Stem Cell. 2017;21:489-501.e7.

22. Schnöder TM, Eberhardt J, Koehler M, Bierhoff HB, Weinert S, Pandey AD, et al. Cell autonomous expression of CXCL-10 in JAK2V617F-mutated MPN. J Cancer Res Clin Oncol. 2017;143:807-20.

23. Manshouri T, Estrov Z, Quintás-Cardama A, Burger J, Zhang Y, Livun A, et al. Bone marrow stroma-secreted cytokines protect JAK2(V617F)-mutated cells from the effects of a JAK2 inhibitor. Cancer Res. 2011;71:3831-40.

24. Majumder S, Zhou LZ-H, Chaturvedi P, Babcock G, Aras S, Ransohoff RM. p48/STAT-1 $\alpha$-containing complexes play a predominant role in induction of IFN- $\gamma$-inducible protein, $10 \mathrm{kDa}$ (IP10) by IFN- $\gamma$ alone or in synergy with TNF- $\alpha$. J Immunol. 1998;161:4736-44.

25. Arber DA, Orazi A, Hasserjian R, Thiele J, Borowitz MJ, Le Beau MM, et al. The2016 revision to the World Health Organization classification of myeloid neoplasms and acute leukemia. Blood. 2016;127:2391-405.

26. Farber JM. A macrophage mRNA selectively induced by gammainterferon encodes a member of the platelet factor 4 family of cytokines. Proc Natl Acad Sci USA. 1990;87:5238-42.

27. Luster AD, Unkeless JC, Ravetch JV. Gamma-interferon transcriptionally regulates an early-response gene containing homology to platelet proteins. Nature. 1985;315:672-6.

28. Algood HMS, Lin PL, Yankura D, Jones A, Chan J, Flynn JL. TNF influences chemokine expression of macrophages in vitro and that of $\mathrm{CD} 11 \mathrm{~b}+$ cells in vivo during Mycobacterium tuberculosis infection. J Immunol Baltim Md 1950. 2004;172:6846-57.

29. Heaton WL, Senina AV, Pomicter AD, Salama ME, Clair PM, Yan D, et al. Autocrine Tnf signaling favors malignant cells in myelofibrosis in a Tnfr2-dependent fashion. Leukemia, 2018. https://doi.org/10.1038/s41375-018-0131-z.

30. Bock O, Loch G, Schade U, von Wasielewski R, Schlué J, Kreipe $\mathrm{H}$. Aberrant expression of transforming growth factor beta-1 (TGF beta-1) per se does not discriminate fibrotic from non-fibrotic chronic myeloproliferative disorders. J Pathol. 2005;205:548-57.

31. Baba T, Tanabe Y, Yoshikawa S, Yamanishi Y, Morishita S, Komatsu N, et al. MIP-1 $\alpha / C C L 3$-expressing basophil-lineage cells drive the leukemic hematopoiesis of chronic myeloid leukemia in mice. Blood. 2016;127:2607-17.

32. Marty C, Lacout C, Droin N, Le Couédic J-P, Ribrag V, Solary E, et al. A role for reactive oxygen species in JAK2 V617F myeloproliferative neoplasm progression. Leukemia. 2013;27:2187-95.

33. Plo I, Nakatake M, Malivert L, Villartay J-P, de, Giraudier S, Villeval J-L, et al. JAK2 stimulates homologous recombination and genetic instability: potential implication in the heterogeneity of myeloproliferative disorders. Blood. 2008;112:1402-12.

34. Chen E, Ahn JS, Sykes DB, Breyfogle LJ, Godfrey AL, Nangalia $\mathrm{J}$, et al. RECQL5 suppresses oncogenic JAK2-induced replication stress and genomic instability. Cell Rep. 2015;13:2345-52.

35. Kozlov SV, Waardenberg AJ, Engholm-Keller K, Arthur JW, Graham ME, Lavin M. Reactive oxygen species (ROS)-activated ATM-dependent phosphorylation of cytoplasmic substrates identified by large-scale phosphoproteomics screen. Mol Cell Proteomics. 2016;15:1032-47.
36. Bennett BT, Bewersdorf J, Knight KL. Immunofluorescence imaging of DNA damage response proteins: optimizing protocols for super-resolution microscopy. Methods San Diego Calif. 2009;48:63-71.

37. Pearl LH, Schierz AC, Ward SE, Al-Lazikani B, Pearl FMG. Therapeutic opportunities within the DNA damage response. Nat Rev Cancer. 2015;15:nrc3891.

38. Hubackova S, Krejcikova K, Bartek J, Hodny Z. IL1- and TGF $\beta$ Nox4 signaling, oxidative stress and DNA damage response are shared features of replicative, oncogene-induced, and druginduced paracrine 'bystander senescence'. Aging. 2012;4:932-51.

39. Chen E, Ahn JS, Massie CE, Clynes D, Godfrey AL, Li J, et al. JAK2V617F promotes replication fork stalling with diseaserestricted impairment of the intra-S checkpoint response. Proc Natl Acad Sci USA. 2014;111:15190-5.

40. Bartek J, Lukas J. Chk1 and Chk2 kinases in checkpoint control and cancer. Cancer Cell. 2003;3:421-9.

41. Harris IS, Treloar AE, Inoue S, Sasaki M, Gorrini C, Lee KC, et al. Glutathione and thioredoxin antioxidant pathways synergize to drive cancer initiation and progression. Cancer Cell. 2015;27:211-22.

42. Lee J, Liu L, Levin DE. Stressing out or stressing in: intracellular pathways for SAPK activation. Curr Genet. 2018. https://doi.org/ 10.1007/s00294-018-0898-5.

43. Arthur JSC, Ley SC. Mitogen-activated protein kinases in innate immunity. Nat Rev Immunol. 2013;13:679-92.

44. Karigane D, Kobayashi H, Morikawa T, Ootomo Y, Sakai M, Nagamatsu G, et al. p38 $\alpha$ activates purine metabolism to initiate hematopoietic stem/progenitor cell cycling in response to stress. Cell Stem Cell. 2016;19:192-204.

45. Iwasa $\mathrm{H}, \mathrm{Han} J$, Ishikawa F. Mitogen-activated protein kinase $\mathrm{p} 38$ defines the common senescence-signalling pathway. Genes Cells Devoted Mol Cell Mech. 2003;8:131-44.

46. Lu M, Zhang W, Li Y, Berenzon D, Wang X, Wang J, et al. Interferon-alpha targets JAK2V617F-positive hematopoietic progenitor cells and acts through the p38 MAPK pathway. Exp Hematol. 2010;38:472-80.

47. Kidger AM, Keyse SM. The regulation of oncogenic Ras/ERK signalling by dual-specificity mitogen activated protein kinase phosphatases (MKPs). Semin Cell Dev Biol. 2016;50:125-32.

48. Bagnyukova TV, Restifo D, Beeharry N, Gabitova L, Li T, Serebriiskii IG, et al. DUSP6 regulates drug sensitivity by modulating DNA damage response. Br J Cancer. 2013;109:1063-71.

49. Kesarwani M, Kincaid Z, Gomaa A, Huber E, Rohrabaugh S, Siddiqui Z, et al. Targeting c-FOS and DUSP1 abrogates intrinsic resistance to tyrosine-kinase inhibitor therapy in BCR-ABLinduced leukemia. Nat Med. 2017;23:472-82.

50. Wernig G, Chen S-Y, Cui L, Van Neste C, Tsai JM, Kambham N, et al. Unifying mechanism for different fibrotic diseases. Proc Natl Acad Sci USA. 2017;114:4757-62.

51. Zhou A, Scoggin S, Gaynor RB, Williams NS. Identification of NF-kappa B-regulated genes induced by TNFalpha utilizing expression profiling and RNA interference. Oncogene. 2003;22:2054-64.

52. Molina G, Vogt A, Bakan A, Dai W, Queiroz de Oliveira P, Znosko W, et al. Zebrafish chemical screening reveals an inhibitor of Dusp6 that expands cardiac cell lineages. Nat Chem Biol. 2009;5:680-7.

53. Bartkova J, Horejsí Z, Koed K, Krämer A, Tort F, Zieger K, et al. DNA damage response as a candidate anti-cancer barrier in early human tumorigenesis. Nature. 2005;434:864-70.

54. Gorgoulis VG, Vassiliou L-VF, Karakaidos $\mathrm{P}$, Zacharatos $\mathrm{P}$, Kotsinas A, Liloglou T, et al. Activation of the DNA damage checkpoint and genomic instability in human precancerous lesions. Nature. 2005;434:907-13. 
55. Bartek J, Bartkova J, Lukas J. DNA damage signalling guards against activated oncogenes and tumour progression. Oncogene. 2007;26:7773-9.

56. Bartkova J, Hamerlik P, Stockhausen M-T, Ehrmann J, Hlobilkova A, Laursen $\mathrm{H}$, et al. Replication stress and oxidative damage contribute to aberrant constitutive activation of DNA damage signalling in human gliomas. Oncogene. 2010;29:5095-102.

57. Rasmussen RD, Gajjar MK, Tuckova L, Jensen KE, MayaMendoza A, Holst CB, et al. BRCA1-regulated RRM2 expression protects glioblastoma cells from endogenous replication stress and promotes tumorigenicity. Nat Commun. 2016;7:13398.

58. Takacova S, Slany R, Bartkova J, Stranecky V, Dolezel P, Luzna $\mathrm{P}$, et al. DNA damage response and inflammatory signaling limit the MLL-ENL-induced leukemogenesis in vivo. Cancer Cell. 2012;21:517-31.

59. Halazonetis TD, Gorgoulis VG, Bartek J. An oncogene-induced DNA damage model for cancer development. Science. 2008;319:1352-5.

60. Cerquozzi S, Tefferi A. Blast transformation and fibrotic progression in polycythemia vera and essential thrombocythemia: a literature review of incidence and risk factors. Blood Cancer J. 2015;5:e366.

61. Wiseman H, Halliwell B. Damage to DNA by reactive oxygen and nitrogen species: role in inflammatory disease and progression to cancer. Biochem J. 1996;313(Pt 1):17-29.

62. Li J, Spensberger D, Ahn JS, Anand S, Beer PA, Ghevaert C, et al. JAK2 V617F impairs hematopoietic stem cell function in a conditional knock-in mouse model of JAK2 V617F-positive essential thrombocythemia. Blood. 2010;116:1528-38.

63. Kamata H, Honda S-I, Maeda S, Chang L, Hirata H, Karin M. Reactive oxygen species promote TNFalpha-induced death and sustained JNK activation by inhibiting MAP kinase phosphatases. Cell. 2005;120:649-61.

64. Vener C, Novembrino C, Bamonti Catena F, Fracchiolla NS, Gianelli U, Savi F, et al. Oxidative stress is increased in primary and post-polycythemia vera myelofibrosis. Exp Hematol. 2010;38:1058-65.

65. Wagner EF, Nebreda AR. Signal integration by JNK and p38 MAPK pathways in cancer development. Nat Rev Cancer. 2009;9:537-49.

66. Hu P, Nebreda AR, Hanenberg H, Kinnebrew GH, Ivan M, Yoder $\mathrm{MC}$, et al. P38 $\alpha / \mathrm{JNK}$ signaling restrains erythropoiesis by suppressing Ezh2-mediated epigenetic silencing of Bim. Nat Commun. 2018;9:3518.

67. Warpman Berglund U, Sanjiv K, Gad H, Kalderén C, Koolmeister T, Pham T, et al. Validation and development of MTH1 inhibitors for treatment of cancer. Ann Oncol. 2016;27:2275-83.

68. Smith C, Abalde-Atristain L, He C, Brodsky BR, Braunstein EM, Chaudhari $\mathrm{P}$, et al. Efficient and allele-specific genome editing of disease loci in human iPSCs. Mol Ther. 2015;23:570-7.
69. Smyth GK. Linear models and empirical bayes methods for assessing differential expression in microarray experiments. Stat Appl Genet Mol Biol. 2004;3:Article 3.

70. Gentleman RC, Carey VJ, Bates DM, Bolstad B, Dettling M, Dudoit S, et al. Bioconductor: open software development for computational biology and bioinformatics. Genome Biol. 2004;5: R80.

71. Carvalho BS, Irizarry RA. A framework for oligonucleotide microarray preprocessing. Bioinforma Oxf Engl. 2010;26:2363-7.

72. Storey JD, Tibshirani R. Statistical significance for genomewide studies. Proc Natl Acad Sci USA. 2003;100:9440-5.

73. Babicki S, Arndt D, Marcu A, Liang Y, Grant JR, Maciejewski A, et al. Heatmapper: web-enabled heat mapping for all. Nucleic Acids Res. 2016;44:W147-W153.

74. Herwig R, Hardt C, Lienhard M, Kamburov A. Analyzing and interpreting genome data at the network level with ConsensusPathDB. Nat Protoc. 2016;11:1889-907.

75. Subramanian A, Tamayo P, Mootha VK, Mukherjee S, Ebert BL, Gillette MA, et al. Gene set enrichment analysis: a knowledgebased approach for interpreting genome-wide expression profiles. Proc Natl Acad Sci USA. 2005;102:15545-50.

76. Horvathova M, Kapralova K, Zidova Z, Dolezal D, Pospisilova D, Divoky V. Erythropoietin-driven signaling ameliorates the survival defect of DMT1-mutant erythroid progenitors and erythroblasts. Haematologica. 2012;97:1480-8.

77. Koledova Z, Kafkova LR, Calabkova L, Krystof V, Dolezel P, Divoky V. Cdk2 inhibition prolongs G1 phase progression in mouse embryonic stem cells. Stem Cells Dev. 2010;19:181-94.

78. Beutler E, Blume KG, Kaplan JC, Löhr GW, Ramot B, Valentine WN. International Committee for Standardization in Haematology: recommended methods for red-cell enzyme analysis*. Br J Haematol. 1977;35:331-40.

79. Zidova Z, Kapralova K, Koralkova P, Mojzikova R, Dolezal D, Divoky V, et al. DMT1-mutant erythrocytes have shortened life span, accelerated glycolysis and increased oxidative stress. Cell Physiol Biochem. 2014;34:2221-31.

80. Andersson A-C, Strömberg S, Bäckvall H, Kampf C, Uhlen M, Wester K, et al. Analysis of protein expression in cell microarrays: a tool for antibody-based proteomics. J Histochem Cytochem. 2006;54:1413-23.

81. van Dekken H, Hop WCJ, Tilanus HW, Haringsma J, van der Valk H, Wink JC, et al. Immunohistochemical evaluation of a panel of tumor cell markers during malignant progression in Barrett esophagus. Am J Clin Pathol. 2008;130:745-53.

82. Ziv Y, Bielopolski D, Galanty Y, Lukas C, Taya Y, Schultz DC, et al. Chromatin relaxation in response to DNA double-strand breaks is modulated by a novel ATM- and KAP-1 dependent pathway. Nat Cell Biol. 2006;8:870-6.

\section{Affiliations}

\section{J. Stetka $\mathbb{1}^{1} \cdot$ P. Vyhlidalova $\mathbb{1}^{1,2} \cdot$ L. Lanikova ${ }^{1,3} \cdot$ P. Koralkova ${ }^{1} \cdot$ J. Gursky $\mathbb{1}^{4} \cdot$ A. Hlusi ${ }^{5} \cdot$ P. Flodr ${ }^{6} \cdot$ S. Hubackova ${ }^{7}$. J. Bartek ${ }^{4,8,9,10} \cdot$ Z. Hodny ${ }^{9}$ V. Divoky ${ }^{1,5}$}

1 Department of Biology, Faculty of Medicine and Dentistry, Palacky University, Olomouc, Czech Republic

2 Department of Histology and Embryology, Faculty of Medicine and Dentistry, Palacky University, Olomouc, Czech Republic

3 Laboratory of Cell and Developmental Biology, Institute of
Molecular Genetics of the ASCR, v. v. i., Prague, Czech Republic

4 Institute of Molecular and Translational Medicine, Faculty of Medicine and Dentistry, Palacky University, Olomouc, Czech Republic

5 Department of Hemato-Oncology, University Hospital and Faculty 
of Medicine and Dentistry, Palacky University, Olomouc, Czech Republic

6 Department of Clinical and Molecular Pathology, University Hospital and Faculty of Medicine and Dentistry, Palacky University, Olomouc, Czech Republic

7 Laboratory of Molecular Therapy, Institute of Biotechnology, BIOCEV, Czech Academy of Sciences, Prague-West 252 50, Czech Republic
8 Danish Cancer Society Research Center, DK-2100 Copenhagen, Denmark

9 Laboratory of Genome Integrity, Institute of Molecular Genetics of the ASCR, v. v. i., Prague, Czech Republic

10 Division of Genome Biology, Department of Biochemistry and Biophysics, Science for Life Laboratory, Karolinska Institute, Stockholm, Sweden 